Article

\title{
Improved OCV Model of a Li-Ion NMC Battery for Online SOC Estimation Using the Extended Kalman Filter
}

\author{
Ines Baccouche ${ }^{1,2, *}$, Sabeur Jemmali ${ }^{1}$, Bilal Manai ${ }^{3}$, Noshin Omar ${ }^{4}$ and \\ Najoua Essoukri Ben Amara ${ }^{1}$ \\ 1 LATIS-Laboratory of Advanced technology and Intelligent Systems, ENISo, Sousse University, BP 526, \\ 4002 Sousse, Tunisia; sabeur.jemmali@aist.enst.fr (S.J.); najoua.benamara@eniso.rnu.tn (N.E.B.A.) \\ 2 ENIM, Monastir University, Ibn El Jazzar 5019, 5035 Monastir, Tunisia \\ 3 IntelliBatteries Company, SoftTech Firm Incubator, Technopole of Sousse, BP 24 Sousse Corniche 4059, \\ 4002 Sousse, Tunisia; bilalmanai@gmail.com \\ 4 MOBI-Mobility, Logistics and Automotive Technology Research Center, Vrije Universiteit Brussel, \\ Pleinlaan 2, 1050 Brussels, Belgium; noshomar@vub.ac.be \\ * Correspondence: ines.baccouche@hotmail.fr; Tel.: +216-21-536-422
}

Academic Editor: Izumi Taniguchi

Received: 22 March 2017; Accepted: 26 May 2017; Published: 31 May 2017

\begin{abstract}
Accurate modeling of the nonlinear relationship between the open circuit voltage (OCV) and the state of charge (SOC) is required for adaptive SOC estimation during the lithium-ion (Li-ion) battery operation. Online SOC estimation should meet several constraints, such as the computational cost, the number of parameters, as well as the accuracy of the model. In this paper, these challenges are considered by proposing an improved simplified and accurate OCV model of a nickel manganese cobalt (NMC) Li-ion battery, based on an empirical analytical characterization approach. In fact, composed of double exponential and simple quadratic functions containing only five parameters, the proposed model accurately follows the experimental curve with a minor fitting error of $1 \mathrm{mV}$. The model is also valid at a wide temperature range and takes into account the voltage hysteresis of the OCV. Using this model in SOC estimation by the extended Kalman filter (EKF) contributes to minimizing the execution time and to reducing the SOC estimation error to only $3 \%$ compared to other existing models where the estimation error is about $5 \%$. Experiments are also performed to prove that the proposed OCV model incorporated in the EKF estimator exhibits good reliability and precision under various loading profiles and temperatures.
\end{abstract}

Keywords: Li-ion batteries; open circuit voltage; battery modeling; battery characterization; state of charge estimation; extended Kalman filter

\section{Introduction}

As a prominent component in the energy field, rechargeable batteries as energy storage systems present a real catalyst for the development of the new power technologies. Indeed, they are considered as the major clean energy sources for most autonomous applications, such as portable devices, smart-grid and electric vehicles (EVs). Among the various choices of rechargeable batteries [1-3], Li-ion batteries can offer many advantages such as portability and compactness given their good characteristics of high energy density, wide voltage range and long cycle lifetime [1,4]. Since the batteries are used to power a wide range of applications, from smartphones to EV, the concern of having a reliable and efficient battery management system (BMS) has increased considerably. BMS has four main functions; namely, balancing, protection, authentication and monitoring [5-7]. When the BMS is mounted on applications like construction gears, electric power tools and light electric 
vehicles (LEV), accurate and simplified modeling of batteries will be essential considering that in these types of applications, complexity, compactness and speed are the key elements. Therefore, making a compromise between an accurate and simple battery model and the precision of the online SOC monitoring is a big challenge raised in embedded systems.

Li-ion battery monitoring is one of the most crucial tasks in BMS, consisting of SOC estimation, commonly defined as the maximum remaining capacity inside the battery. In fact, SOC serves as a fuel gauging that controls the use of the device equipped with a battery and subsequently controls its performance and efficiency.

Many studies about battery SOC estimation have been reported in the literature [8,9]. The electrochemical methods are shown to be highly accurate, but invasive and destructive, since they need access to internal electrochemical quantities, like lithium ions' dimensions and electrolyte concentration $[10,11]$. Furthermore, empirical methods are used, where the battery is considered as a black-box without knowing the internal behavior based on artificial intelligence algorithms like neural networks and fuzzy logic algorithms [12,13]. Their effectiveness depends on the accuracy of the learning data, and they are costly in terms of processing and time consumption [14]. Another category of methods is book-keeping; its principle is to count the amount of charge available in the battery $[8,14,15]$. This Coulomb-counting method is based on the battery current integration over time; thus, it is simple to implement and gives an acceptable precision, but suffers from cumulative errors due to the measurements inaccuracies $[16,17]$. Moreover, the model-based methods are increasingly used for SOC estimation, they are reported as accurate and suitable for online estimation since they consist of combing an equivalent battery model and an adaptive state-observer algorithm [11,15,18-20]. These methods' reliability is linked to the accuracy of the model, so understanding the battery behaviors is of great importance [21].

Online estimation of SOC during system operation requires the development of a sufficiently simplified, but adequately accurate battery model that can describe the battery behavior in terms of its chemical, electrical and thermal aspects [22]. Reviewing the literature concerning the modeling of Li-ion batteries' behavior, it is found that multiple models have been developed and used for different applications based on the battery system [23]. Many approaches of modeling are highlighted and can be classified as electrochemical models, thermal models, electrical models and interdisciplinary models [23-26]. According to the literature, the electrical models, among them the equivalent-circuit models, are the most used because they create a link between some real physical phenomena inside the battery and the electrical component of the model [25]. The best known model is the basic Rint model composed of a voltage source and an internal resistance introduced in [25]. This model was upgraded to a battery model-based on resistor-capacitor (RC) branches to display the diffusion phenomena of the Li-ion battery. We found then the first-order model-based on a unique RC branch, the second-order model with two RCs and $n$-th-order model as cited in [27] in which the number of RCs can be multiplied for very highly accurate modeling. In addition, the complexity of the model should be carefully considered when it is meant to be applied in real-time systems.

The model-based approaches need an accurate open circuit voltage (OCV) model, which connects OCV to SOC. The OCV modeling can be either numerical, as a look-up table, or analytical, as a mathematical expression; however, the latter has some advantages including computational effectiveness since no interpolation is required like linear regression or spline algorithms [28]. Nevertheless, the OCV curve generally shows a hysteresis even if it is not really considerable at the early life of the battery, this phenomenon is clearly revealed during the battery life cycles. This gap between the charge and discharge curves can be restrictive when it comes to estimating SOC [29,30]. Indeed, a minor mismatch in OCV curve fitting can cause a wide deviation in SOC estimation. Therefore, adjustment of the OCV models can give a significant contribution to increase the accuracy of SOC estimation [22,31-33].

In this paper, we propose an improved OCV-SOC model injected in a Thevenin first-order battery model, which is chosen since it is reported in many works [23] to be sufficiently adequate for online 
estimation. The proposed model is then used with the Kalman filter as an adaptive state observer algorithm to estimate the SOC. The proposed model has the advantage of being composed from simplified functions and less coefficients taking into account the hysteresis. This contribution has shown an improvement in the accuracy of the SOC estimation comparing with other existing OCV-SOC models in the literature $[22,34,35]$. The study is based on a battery life cycle test charge/discharge of a nickel manganese cobalt oxide (NMC) pouch battery of $20 \mathrm{Ah}$ as a nominal capacity performed at a wide margin of temperatures.

The remainder of the paper is organized as follows. In the next section, an overview of the existing OCV models is presented, then we explore the development and parametrization of the proposed OCV model and compare its precision with existing models in the literature. In the third section, we use the improved OCV model for SOC estimation based on EKF where the algorithm principle is well explained. A discussion of the experimental results is then put forward. The final section is dedicated to the conclusion and perspectives.

\section{Open Circuit Voltage Model}

\subsection{Overview of Li-Ion OCV Models}

Battery modeling is an important research field because of their expanding use and the need to reproduce and simulate the physical behaviors of the battery. Much research is being carried in modeling, so that we can have accurate monitoring of Li-ion batteries dynamically. Researchers of batteries have developed a wide range of battery models with varying degrees of complexity. Therefore, to consider the real-time applications, the first-order model is utilized in this paper, which has been widely used for its good characteristics in terms of accuracy and complexity $[26,36,37]$. This battery model is composed of a voltage source representing the open circuit voltage, an ohmic resistance $R_{i}$ that models the internal resistance and a one polarization RC network that describes the polarization phenomena.The $\mathrm{RC}$ branch is composed of the capacitance $C_{d f}$, representing the polarization of the metallic electrodes, and the resistor $R_{d f}$, which is due to the contact of the electrodes with the electrolyte [25]. This model is given by Figure 1, where $I_{b a t}$ is the battery charge or discharge current and $V_{b a t}$ is the battery terminal voltage.

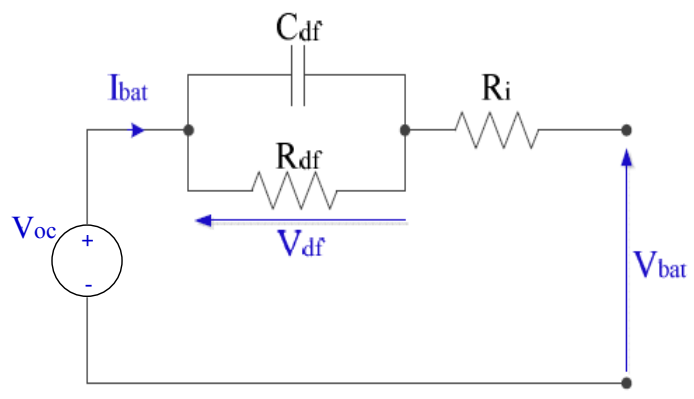

Figure 1. The first-order battery model.

The modeling of the OCV is imperative since it is the main component of the battery model, and it is part of the complex physical behavior during the intercalation and de-intercalation processes of the lithium ions. The OCV of a battery cell is the potential difference between the positive and negative electrodes when no load is applied and the battery is at equilibrium. Several OCV models were proposed in the literature. Some of these models are very simple and do not take into account the nonlinearity, such as the linear approximation, which was upgraded to a piecewise linear approach that increases the accuracy, but still not considering the phenomenological aspect, even if it is easy to implement [15,38]. 
Nevertheless, other methods tried to get as close as possible to the real behavior and to respect the observed plateaus from the experimental curve. The given OCV equations from the experimental data are multiple, as revealed in the literature review as presented in Table 1.

Table 1. Overview of OCV models.

\begin{tabular}{lcc}
\hline & OCV Model Functions & Ref. \\
\hline Polynomial & $O C V(x)=p_{1} x+p_{0}$ & {$[15]$} \\
Logarithmic & $O C V(x)=p_{n} x^{n}+p_{n-1} x^{n-1}+\cdots+p_{1} x+p_{0}$ & {$[34,39]$} \\
Exponential & $O C V(x)=p_{0}+p_{1} \ln \left(\frac{x}{1-x}\right)-p_{2} x$ \\
Combined & $O C V(x)=p_{1} e^{\alpha_{1} x}+p_{0}$ \\
& $O C V(x)=p_{0}+p_{1} \frac{1}{1+e^{\alpha_{1}\left(x-\beta_{1}\right)}}+p_{2} \frac{1}{1+e^{\alpha_{2}\left(x-\beta_{2}\right)}}+p_{3} \frac{1}{1+e^{\alpha_{3}(x-1)}}+p_{4} \frac{1}{1+e^{\alpha_{4} x}}+p_{5}$ \\
& $O C V(x)=p_{0}+p_{1} x+p_{2} x^{2}+p_{3} x^{3}+e^{-\alpha_{1} x}$
\end{tabular}

Some of them were complex, which makes them hard to implement in real-time applications. Polynomial models are among the most used models where the order may reach nine [34,43]. The greater the order of the polynomial, the more precise the model is, but the harder it is to implement in real-time systems. Some other models are proposed based on combining exponential and logarithmic functions $[20,22,34]$, and they are said to be the most accurate. In fact, the Weng model presented in [22] is proven to be more accurate than polynomial models and tracks the phenomenological aspect of the battery, but it presents a great number of parameters. The aim of this work is to propose an OCV model of the Li-ion battery that gives a reliable accuracy and reduces the complexity and the memory consumption in order to be embedded in the BMS of the LEV, hence to monitor the SOC in real-time.

\subsection{Battery Model Characterization}

The studied cell chemistry is a pouch NMC 20-Ah lithium ion with a nominal voltage of $3.65 \mathrm{~V}$, which is dedicated to be used on the BEV especially the LEV battery pack [23,44,45]. All tests have been executed at a wide temperature range $\left(5,15,25,45^{\circ} \mathrm{C}\right)$ so as to validate the model at cold, normal and hot conditions to which the battery pack may be exposed. The battery model created is based on parameters calculated from characterization. To extract the parameters, a series of characterization tests is needed, and this according to many standards and research works [23]. At first, the capacity test is for knowing the effective nominal capacity of the battery, which is given by a standard charge and discharge test.

The second test used is the OCV test, which needs to be done in order to extract the OCV-SOC relationship, as well as the battery model parameters instead of using the hybrid pulse power characterization (HPPC) test, since in this study, we consider only the standard C-rate [46]. The experimental OCV discharge test is given in Figure 2.

Actually, the OCV test consists firstly of the complete charge of the battery, then we apply discharging current pulses corresponding to $5 \%$ of the SOC step intercepted by a 30-min rest time to fully discharge the cell. The rest time is crucial because it allows the battery to reach the equilibrium at a stable value of OCV. Then, we extract the OCV points at the 21 different SOC levels to trace the curve mapping between OCV and SOC meant to be modeled analytically. This setup is then repeated in the charge phase. 


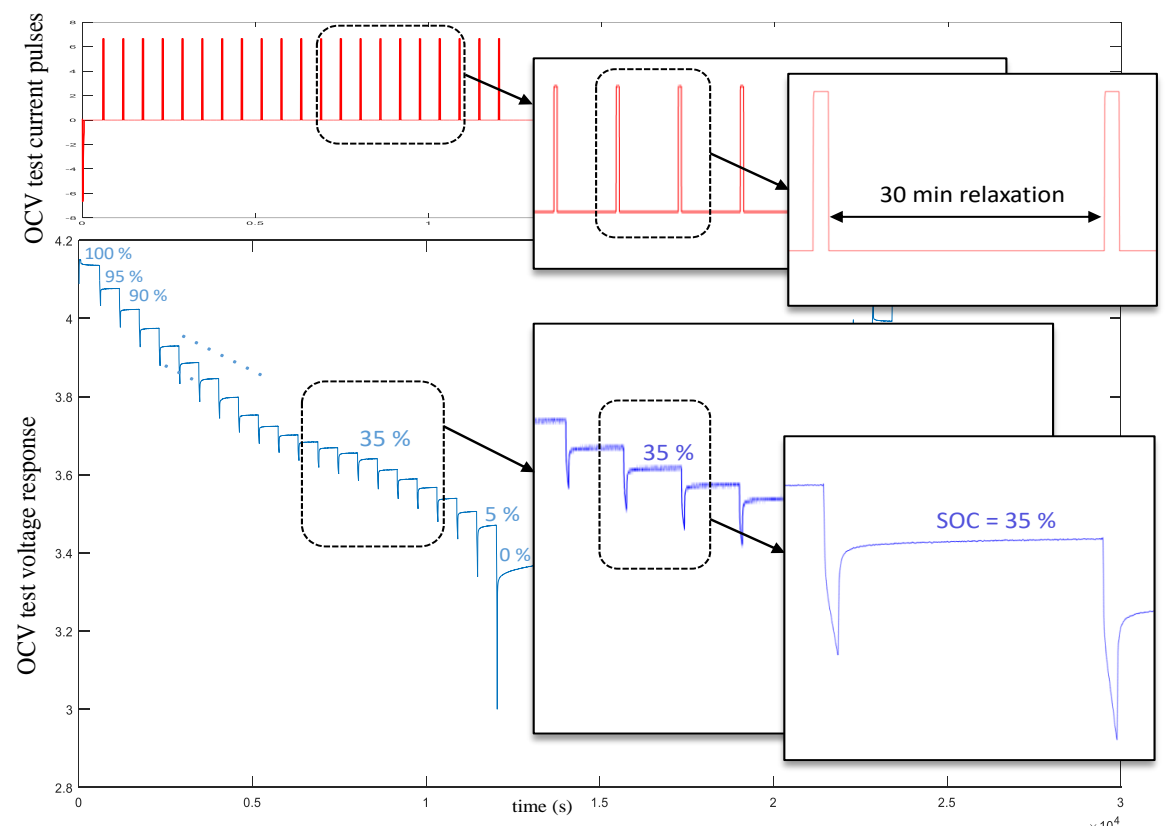

Figure 2. The OCV discharge test current and voltage response.

The voltage response of each current pulse gives the parameters of the battery model at each SOC level. A schematic of the terminal voltage measurements during one complete pulse of the discharge test is shown in Figure 3.

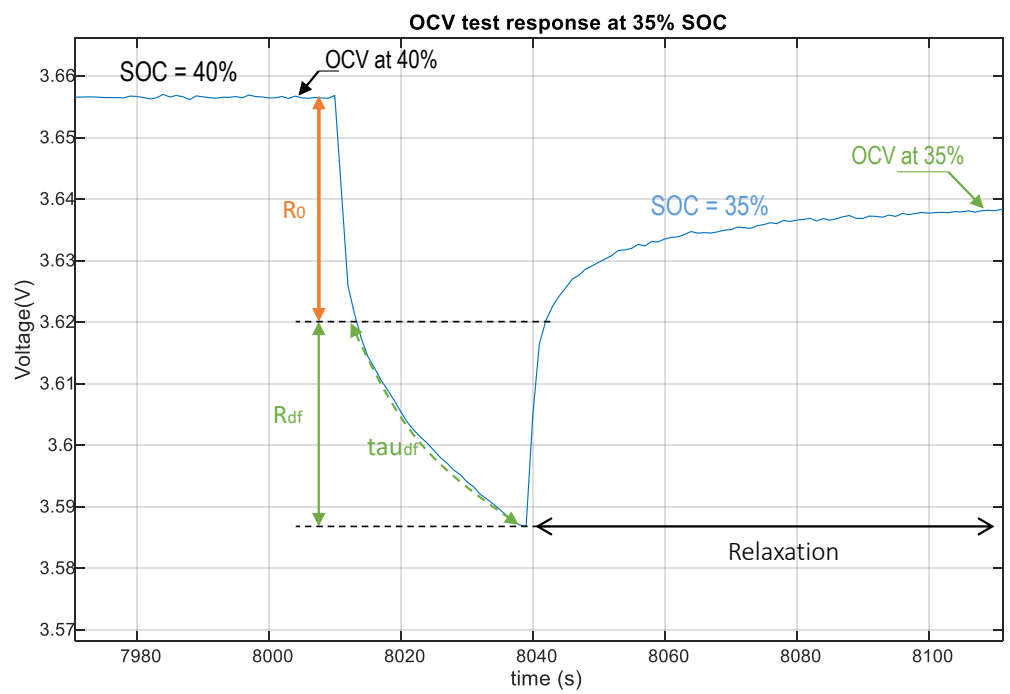

Figure 3. Parameters extraction form OCV test at SOC $=35 \%$.

According to this figure, the fast voltage drop corresponds to the instantaneous response, which is correlated with the internal resistance $\mathrm{R} 0$ of the cell [46]. Otherwise, the observed delayed response corresponding to the time constant tau of the RC network models the diffusion of lithium ions. The extraction procedure is then repeated for each pulse response to extract the first-order model parameters as demonstrated in Figure 3. However, the detected OCV points in the charge and discharge phases, as illustrated in Figure 4, are not exactly superimposed. 


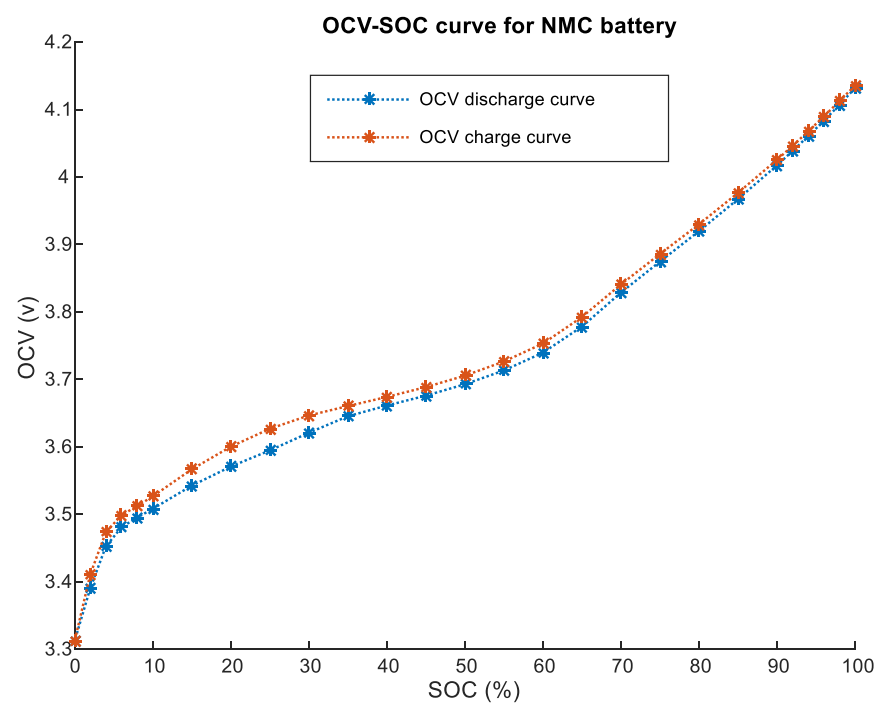

Figure 4. Experimental SOC-OCV curves at $25^{\circ} \mathrm{C}$.

Indeed, the OCV curve presents a hysteresis that is well reported in the literature [30,46-48]. The OCV hysteresis is caused by the lack of relaxation between charging and discharging and the diffusion phenomenon of the transition of the active material during charging and discharging. This gap between the OCV curves may lead to an increase in the error of the SOC estimation after that. Thus, it has be to taken into account in the battery model.

The temperature is another parameter that needs to be considered. In fact, the OCV curve is affected by it, as illustrated in Figure 5, where the effect of the temperature can be visibly distinguished in the SOC margin between $0 \%$ and $20 \%$.

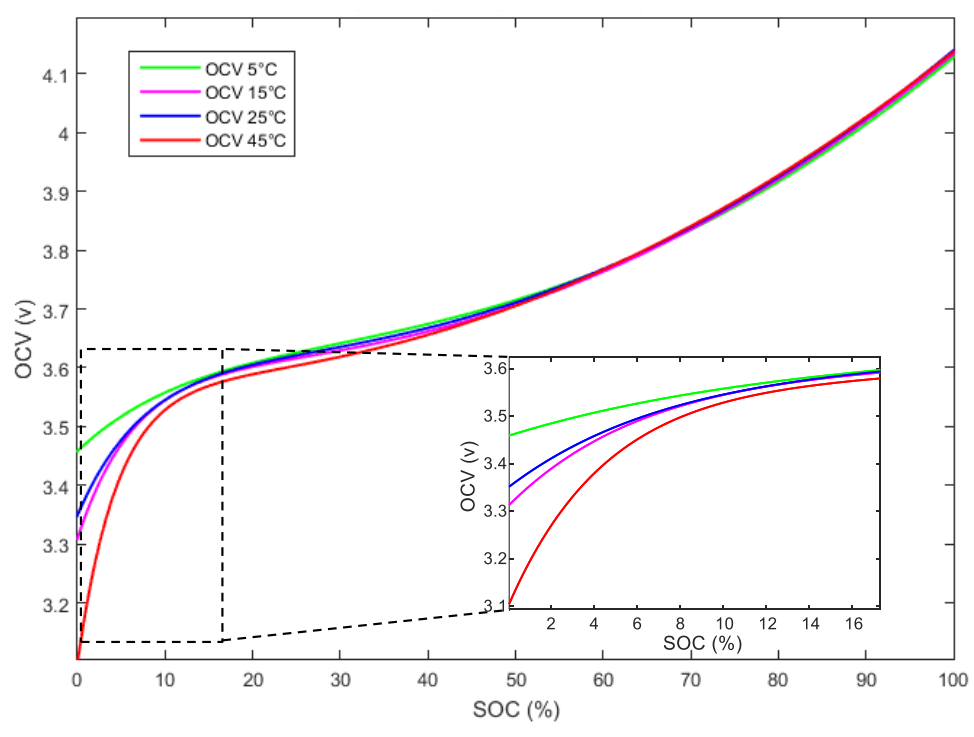

Figure 5. Experimental OCV curves of the charge at different temperatures.

The Thevenin first-order model parameters $R_{i}, R_{d f}$ and $\tau_{d f}$ are approximated to constant values since their observed variation is not extremely considerable, although this consideration contributes to simplifying the model for an online use and an eventual implementation. Thus, the extracted parameters of the charge at different temperatures are available in Table 2. 
Table 2. The extracted parameters of the battery model of the charge at different temperatures.

\begin{tabular}{ccccc}
\hline First-Order Model Parameters & $\mathbf{5}^{\circ}$ & $\mathbf{1 5}^{\circ}$ & $\mathbf{2 5}^{\circ}$ & $\mathbf{4 5}^{\circ}$ \\
\hline$C_{\text {nom }}(\mathrm{Ah})$ & 17.17 & 19.24 & 20 & 21.6 \\
$R_{i}(\Omega)$ & 0.007 & 0.0047 & 0.003 & 0.0019 \\
$R_{d f}(\Omega)$ & 0.0042 & 0.0018 & 0.00065 & 0.00054 \\
$\tau_{d f}(\mathrm{~s})$ & 21 & 19.3 & 16.5 & 15.2 \\
\hline
\end{tabular}

Another test is then needed for the validation, which is the dynamic discharge pulse (DDP) test [23]. The DDP test consists of a series of charge and discharge pulses applied on the battery until the cut-off voltage. It is created to evaluate the effectiveness of the battery model during the dynamic solicitation. Figure 6 illustrates the DDP test current profile and its voltage response, which represents the dynamic discharge behavior.
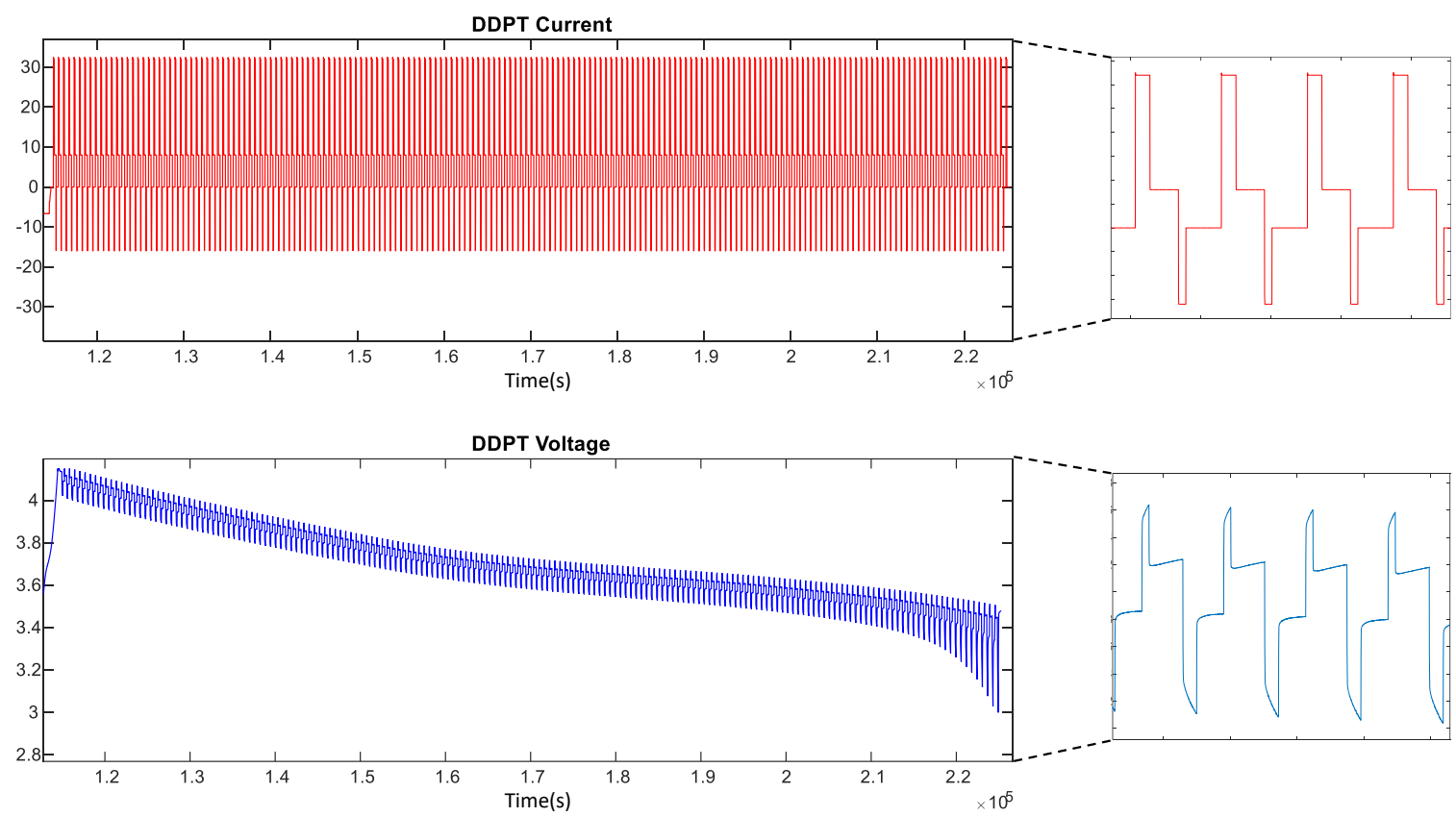

Figure 6. The DDP test.

The extracted parameters in charge and discharge are used in the battery model, and the OCV experimental points were stored as a look-up table. The model was validated in MATLAB-Simulink; thereafter, the obtained simulated voltage and the real battery response of the DDP test were compared.

Figure 7 gives the Simulink schematic of the battery model used for validation; in this model, all of the parameters are divided into charge and discharge to capture the hysteresis.

The result given by the scope representing the precision of the battery model is illustrated in Figure 8.

According to Figure 8 , at $25^{\circ} \mathrm{C}$, the error between the simulated and the real voltage response is almost $1 \%$, but it reaches $5 \%$ when the battery is in the last discharge phase. This increase in error is due to the fact that we have considered tau as a constant, but this still makes the battery model sufficiently reliable to be used for SOC estimation since that of the error of reproducing the terminal voltage is about $1 \%$ in the SOC range between $100 \%$ and $10 \%$. The model used is validated with the dynamic discharge pulse test (DDPT) at various temperatures. 


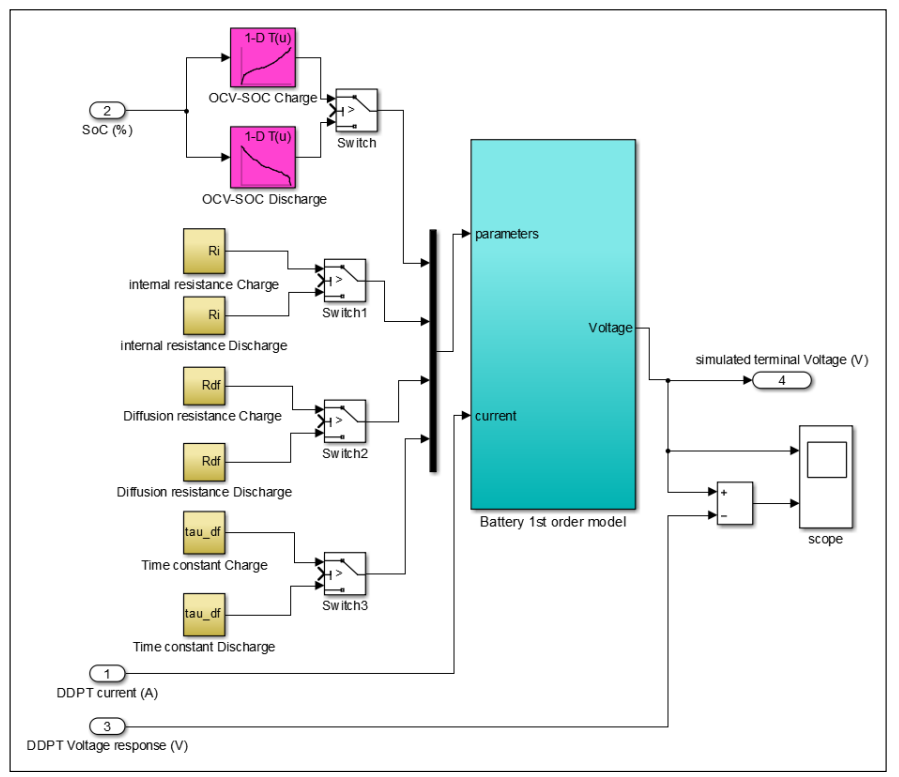

Figure 7. Schematic of the Simulink battery model.
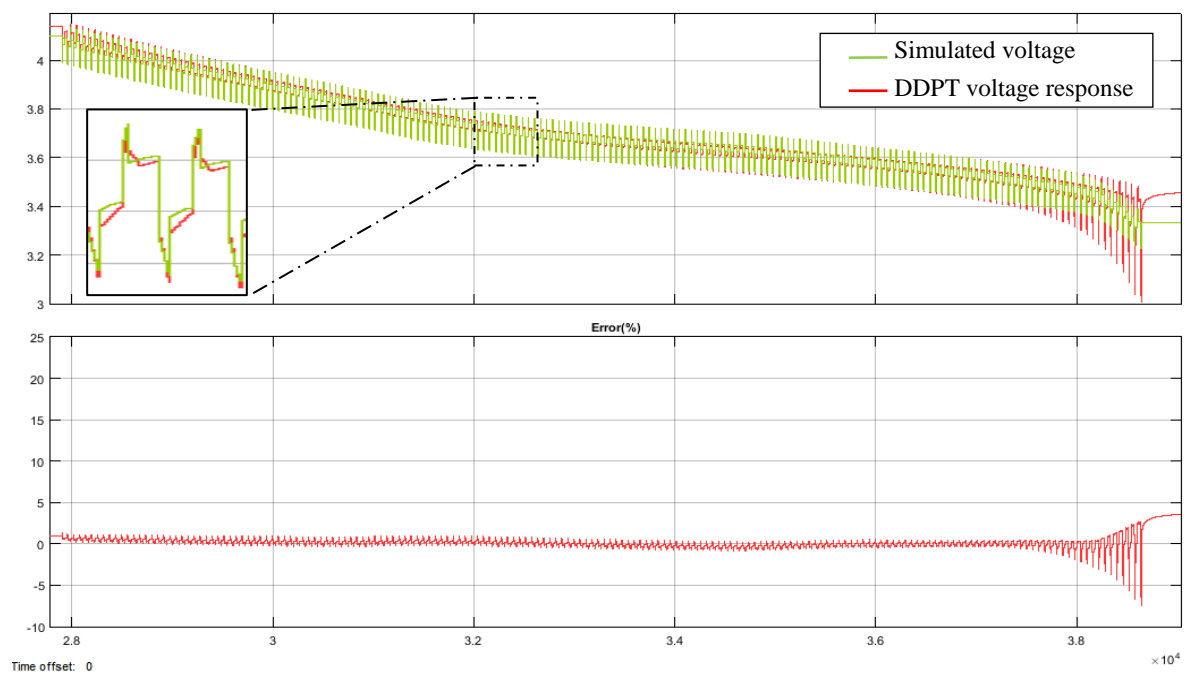

Figure 8. Schematic of the Simulink battery model.

The model has shown its effectiveness even with other temperatures, and the error has not exceeded $1.5 \%$, as seen in Table 3 .

Table 3. Model error at different $\mathrm{T}^{\circ}$ in the SOC window $[100 \%-10 \%]$.

\begin{tabular}{ccccc}
\hline & $5{ }^{\circ} \mathrm{C}$ & $\mathbf{1 5}{ }^{\circ} \mathrm{C}$ & $25{ }^{\circ} \mathrm{C}$ & $45{ }^{\circ} \mathrm{C}$ \\
\hline Error & $\pm 1.5 \%$ & $\pm 1.5 \%$ & $\pm 1 \%$ & $\pm 1.25 \%$ \\
\hline
\end{tabular}

\subsection{Proposed OCV Model}

Instead of using a look-up table, a new OCV model is proposed. It is able to capture the phenomenological aspect associated with the diffusion process while fitting the OCV-SOC data. This model structure is chosen to enforce the model accuracy and also to be implemented for an online SOC estimation with the EKF algorithm. Since our concern is to have an accurate model suitable 
for the embedded BMS of LEV, the reflection was to have a reliable and accurate model, but is also optimized in terms of complexity and memory.

Therefore, we used a combined empirical analytic approach, in order to create a model that not only fits closely the shape of the OCV curve, but that is also linked to the physical diffusion behavior of the battery where the delay could be modeled by an exponential function.

At first, we proposed the simplified double exponential model as given by Equation (1).

$$
O C V(S O C)=p_{1} e^{\left(\alpha_{1} S O C\right)}+p_{2} e^{\left(\alpha_{2} S O C\right)}
$$

The fitting root mean square error (RMSE) was high compared to the other existing models like the polynomial models and the Weng model. Thus to remedy this deficiency, we combined the simplified double exponential with a quadratic term that allows fitting the curve deviation under $40 \%$ of the SOC.

Our proposed OCV-SOC relationship is then defined by Equation (2).

$$
O C V(S O C)=p_{1} e^{\left(\alpha_{1} S O C\right)}+p_{2} e^{\left(\alpha_{2} S O C\right)}+p_{3} S O C^{2}
$$

The proposed OCV model is compared in this section with the four different other models summarized in Table 4. The fitted OCV-SOC curves are plotted in Figure 9.

Table 4. Summary of the studied OCV models.

\begin{tabular}{ccc}
\hline Model & Equations OCV (x) & Coefficient's Number \\
\hline 1. Linear & $p_{1} x+p_{0}$ & 2 \\
2. Polynomial 6deg & $p_{6} x^{6}+p_{5} x^{5}+p_{4} x^{4}+p_{3} x^{3}+p_{2} x^{2}+p_{1} x+p_{0}$ & 7 \\
3. Weng model [22] & $p_{0}+p_{1} \frac{1}{1+e^{\alpha_{1}\left(x-\beta_{1}\right)}}+p_{2} \frac{1}{1+e^{\alpha_{2}\left(x-\beta_{2}\right)}}+p_{3} \frac{1}{1+e^{\alpha_{3}(x-1)}}+p_{4} \frac{1}{1+e^{\alpha_{4} x}}+p_{5} x$ & 12 \\
4. Double exponential & $p_{1} e^{\left(\alpha_{1} x\right)}+p_{2} e^{\left(\alpha_{2} x\right)}$ & 4 \\
5. Proposed model & $p_{1} e^{\left(\alpha_{1} x\right)}+p_{2} e^{\left(\alpha_{2} x\right)}+p_{3} x^{2}$ & 5 \\
\hline
\end{tabular}

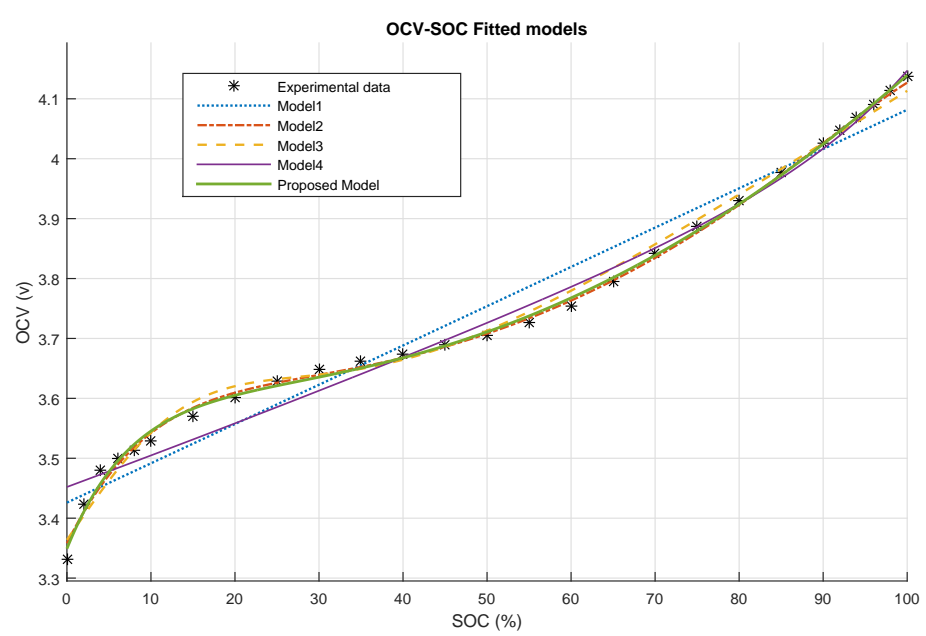

Figure 9. OCV fitting results at $25^{\circ}$ for the charge.

All of the parameters of the studied models were refitted by the MATLAB curve fitting toolbox, and all of the values are available in Table 5 . 
Table 5. The parameters of the fitted OCV models.

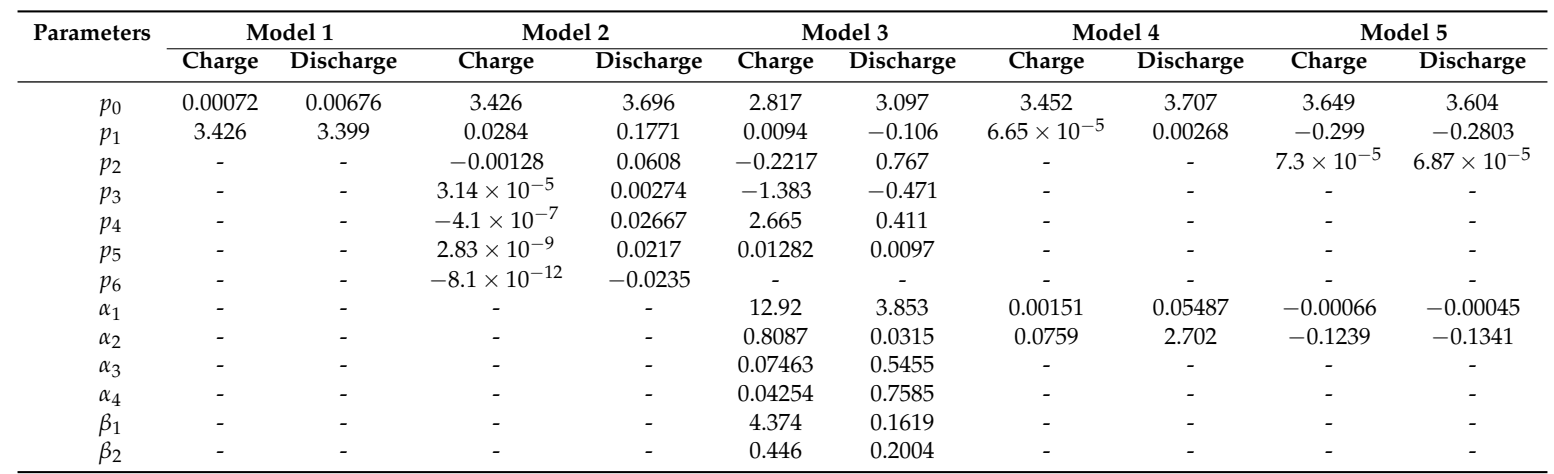

In [34], the polynomial model was reported as the best fit, but the Weng model was then proposed as a more accurate model [22]. One can see in Table 5 that our new model proposed in Equation (3) has a low RMSE value so it presents the best fitting accuracy compared to the studied models, and this is well proven in Figure 9, where the fitted curve of our model is quite close to the experimental data.

Table 6 gives the RMS error of each model at $25^{\circ} \mathrm{C}$, and it is clear that our proposed model is the most precise.

Table 6. Comparison of the OCV models' precision at $25^{\circ} \mathrm{C}$.

\begin{tabular}{cccccc}
\hline Performances & Model 1 & Model 2 & Model 3 & Model 4 & Model 5 \\
\hline RMSE & 0.04274 & 0.01127 & 0.02281 & 0.03449 & 0.01053 \\
R-squared & $96.77 \%$ & $99.80 \%$ & $99.44 \%$ & $98.04 \%$ & $99.84 \%$ \\
\hline
\end{tabular}

Fitting the proposed model to OCV data recorded from the NMC 20-Ah battery cell at different temperatures $\left(5,15,25,45^{\circ} \mathrm{C}\right)$ shows high accuracy, as illustrated in Figure 10.
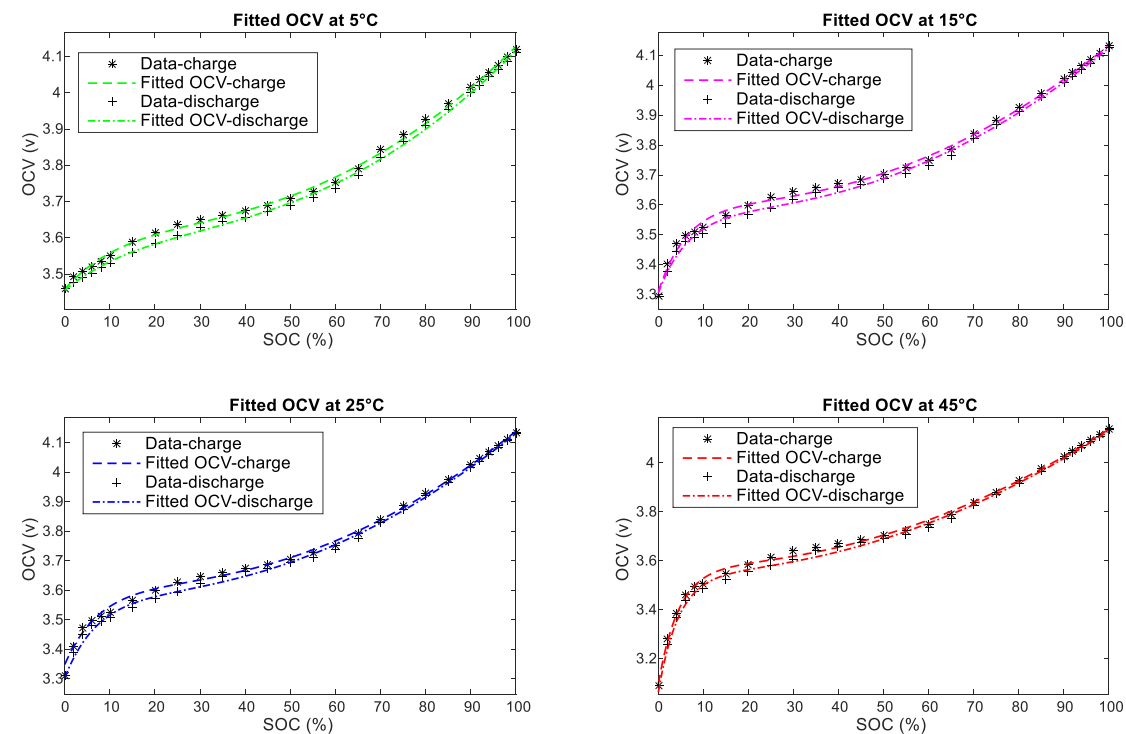

Figure 10. OCV fitting results of the proposed model at different temperatures in charge and discharge.

All of the fitted parameters of the proposed model are presented in Table 7. 
Table 7. Parameters of the proposed fitted OCV model at different temperatures.

\begin{tabular}{ccccccccc}
\hline \multirow{2}{*}{ Parameters } & \multicolumn{2}{c}{$\mathbf{5}{ }^{\circ} \mathbf{C}$} & \multicolumn{2}{c}{$\mathbf{1 5}{ }^{\circ} \mathbf{C}$} & $\mathbf{2 5}^{\circ} \mathbf{C}$ & \multicolumn{4}{c}{${ }^{\circ} \mathbf{C}$} \\
\cline { 2 - 8 } & Charge & Discharge & Charge & Discharge & Charge & Discharge & Charge & Discharge \\
\hline$p_{0}$ & 3.734 & 3.804 & 3.629 & 3.599 & 3.637 & 3.604 & 3.584 \\
$p_{1}$ & -0.2756 & -0.3487 & -0.3191 & -0.2933 & -0.3091 & -0.2803 & -0.4868 \\
$p_{2}$ & $8.013 \times 10^{-5}$ & $8.838 \times 10^{-5}$ & $6.952 \times 10^{-5}$ & $6.9 \times 10^{-5}$ & $7.033 \times 10^{-5}$ & $6.871 \times 10^{-5}$ & $6.212 \times 10^{-5}$ & -0.4573 \\
$\alpha_{1}$ & -0.001155 & -0.001618 & -0.0005411 & -0.0004687 & -0.0005747 & -0.0004523 & -0.0001919 & $-6.241 \times 10^{-5}$ \\
$\alpha_{2}$ & -0.06674 & -0.04724 & -0.1493 & -0.1434 & -0.1366 & -0.1341 & -0.2181 & -0.221 \\
\hline
\end{tabular}

The obtained parameters of the OCV model at the characterization temperatures should be interpolated to be used in intermediate temperatures values. In Figure 11, we have considered a linear interpolation for each parameter of the model at the four known temperatures $\left(5^{\circ}, 15^{\circ}, 25^{\circ}\right.$ and $45^{\circ}$ ) in both charge and discharge. Therefore, the model for other unknown temperatures outside of the characterization ones can be given by a simple projection. The dependencies of all of the model parameters on the temperature are illustrated in Figure 11.
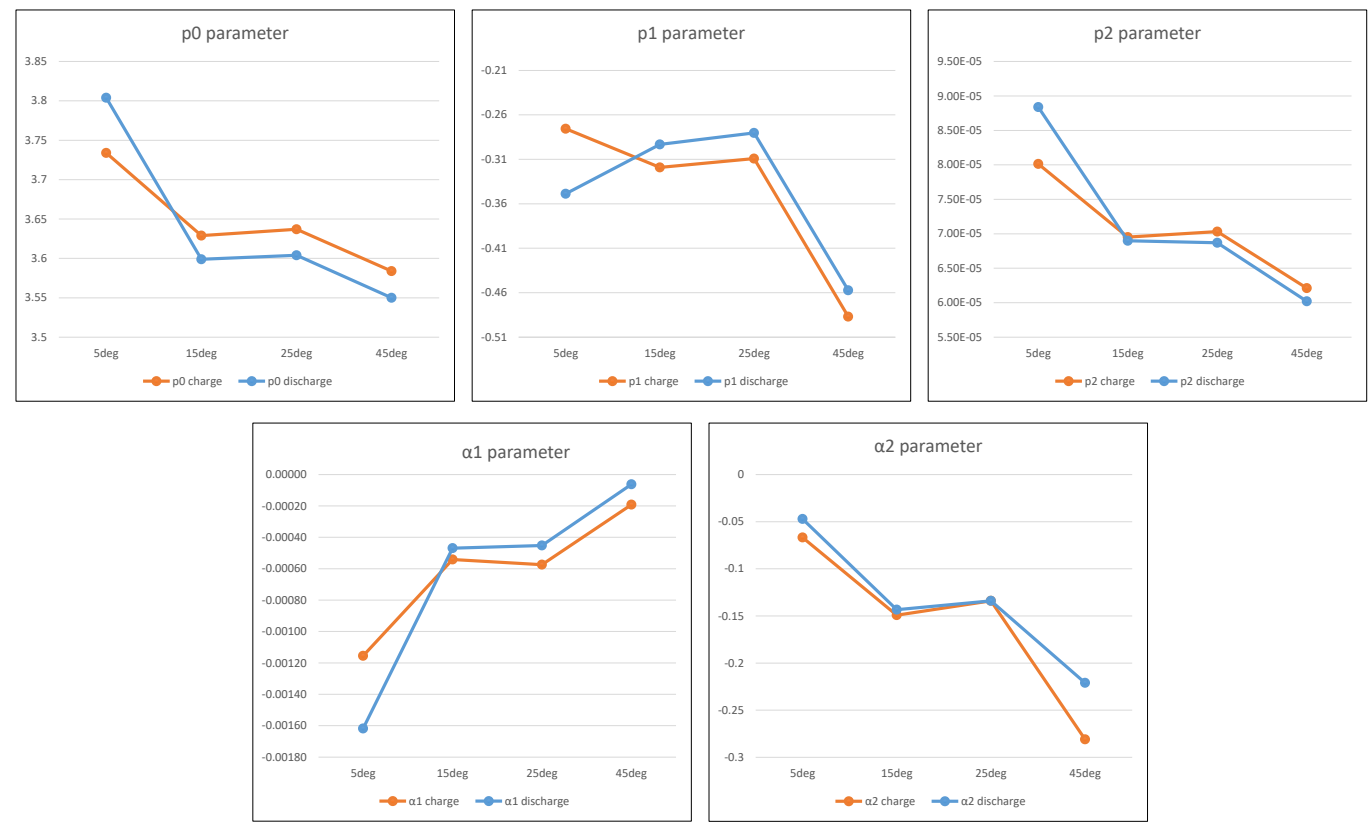

Figure 11. The linear interpolation of the proposed OCV model for the $\left[5-45^{\circ} \mathrm{C}\right]$ temperature range.

Otherwise, the RMS error of the proposed model at the four studied temperatures is around $1 \mathrm{mV}$, as seen in Table 8, which proves the reliability of our model.

Table 8. Comparison of the OCV models' precision at various temperatures.

\begin{tabular}{ccccc}
\hline Performances & $\mathbf{5}^{\circ} \mathbf{C}$ & $\mathbf{1 5}{ }^{\circ} \mathbf{C}$ & $\mathbf{2 5}^{\circ} \mathbf{C}$ & $\mathbf{4 5}{ }^{\circ} \mathbf{C}$ \\
\hline RMSE Charge & 0.0079 & 0.0107 & 0.01053 & 0.01186 \\
RMSE Discharge & 0.00739 & 0.01076 & 0.00982 & 0.0117 \\
\hline
\end{tabular}

Consequently, incorporating the new OCV model in the battery model may also improve the SOC estimation results, which will be demonstrated in the next section. 


\section{State of Charge Monitoring by the Extended Kalman Filter}

\subsection{Application}

The LEV battery packs are composed of a number of assembled cells either in series or in parallel and an electronic card stuck on, serving as a BMS. For example, for an electric scooter, the battery pack has a power supply voltage equal to $36 \mathrm{~V}$ and a capacity of $20 \mathrm{Ah}$, so the battery pack is composed of 10 studied NMC 20-Ah cells mounted in series. The goal of our work is to have an online SOC estimation for this LEV, not too consuming in terms of computation and memory, in order to be adequate for implementation on a hardware platform to be embedded in the BMS, but without sacrificing accuracy. Indeed, for the estimation of the SOC, we use a model-based approach. This approach is based on the first-order equivalent model, which has ensured a good accuracy with the least parameters. In this model, we used as OCV our proposed model, as explained in the previous section. Then, an adaptive method based on a state observation, which is the extended Kalman filter, was used for the SOC estimation, as summarized in Figure 12. In this figure, we distinguish two off-line phases, which are the characterization tests and the modeling phase. The outputs of these tests are then used by the online process consisting of the SOC estimation with EKF, where the model is implemented, and the control input is the current load profile representing the behavior of the scooter engine either in acceleration or deceleration. The output the online process is the SOC value estimated by the EKF.

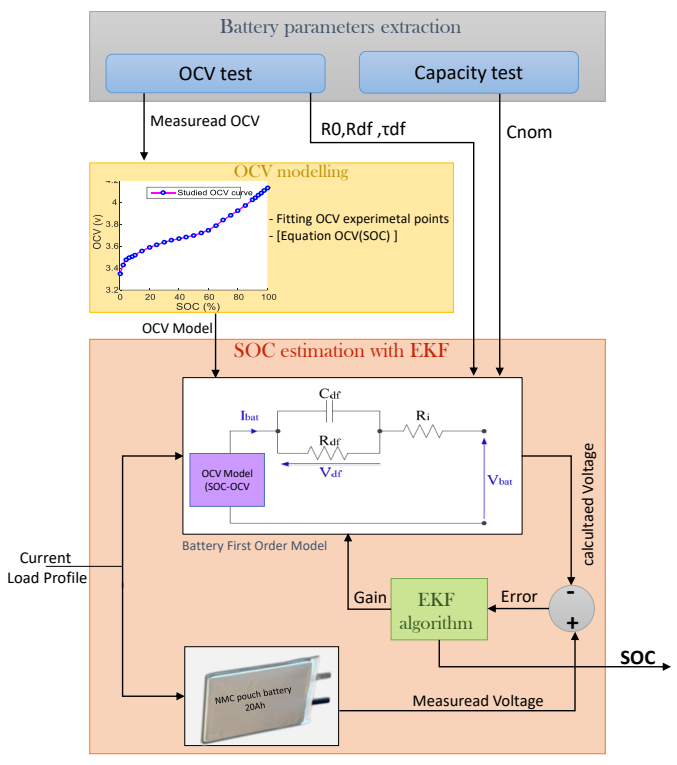

Figure 12. Flowchart of the approach used.

\subsection{The Principle of EKF}

Kalman filtering is an adaptive technique consisting of estimating a nonlinear dynamical system state, used in several fields for the purpose of tracking, navigation and control [8]. It actually uses the dynamics of the system defining its evolution over time to obtain better data, thus eliminating the effect of noise. This estimation technique has been widely used for monitoring Li-ion batteries because they exhibit nonlinear behavior [38,49]. Different variants of Kalman filtering techniques like the linear Kalman filter (KF) [38], the extended Kalman filter (EKF) [35], the unscented Kalman filter (UKF) [50] and the sigma-point Kalman filter (SPKF) [51] have been used to estimate the SOC based on different battery equivalent models. Unlike KF where the system is linearly formulated, the EKF requires a local linearization that consists of using the Jacobian function to bring the system to the conventional form, with interdependent variables rather than a constant matrix [52].

The framework of our discrete-time nonlinear system with noise input is given by Equations (3) and (4). 
The discrete state function of a system can be represented by a state vector $\mathrm{X}$ and a measurement vector $\mathrm{Y}$.

$$
\left\{\begin{array}{l}
X_{k}=A X_{k-1}+B U+w \\
Y_{k}=h\left(X_{k}\right)+D U+v
\end{array}\right.
$$

with:

A: Transition matrix relating the state $\mathrm{k}$ to the state $\mathrm{k}+1$

$\mathrm{U}$ : The command vector

B: Control matrix related to the command $\mathrm{U}$

$\mathrm{w}$ : The perturbation vector related to the state $\mathrm{X}$

$\mathrm{h}$ : Measure function

$\mathrm{v}$ : The measure perturbation

Algorithm 1 resumes the steps of the EKF used based basically on two major phases, the prediction based on previous states and the correction based on updating data by the measurement.

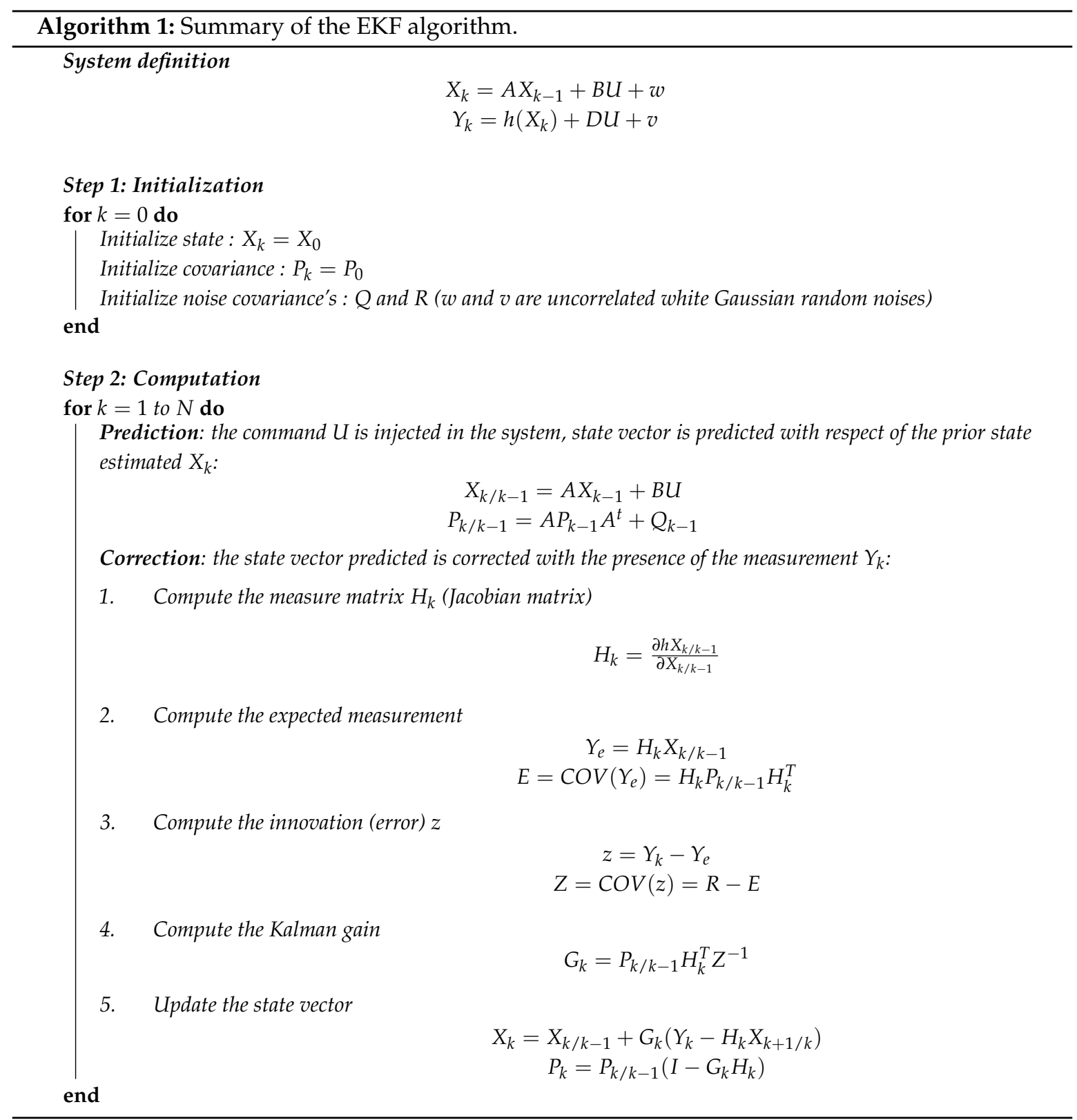


The system input is the command $\mathrm{U}$, which is known and measurable. However, the measurement could result in errors caused by the stochastic noise, $\mathrm{w}$, which cannot be measured and which affects the state of the system. The system output $Y_{k}$ is expressed in the output equation in terms of a state vector function, the command and noise caused by the measurement process. Furthermore, $\mathrm{X}, \mathrm{w}$ and $\mathrm{v}$ are non-measurable and may contain uncertainties, so to quantify them, we associate respectively $P, Q$ and $\mathrm{R}$, which are their associated covariance matrix to be used then in the EKF operation.

Thus, the connection of the first-order battery model to the extended Kalman filter with respect to Algorithm 1 is illustrated in Figure 13. All blocks are then explained, and the analogy between EKF terms and the battery model is stated.

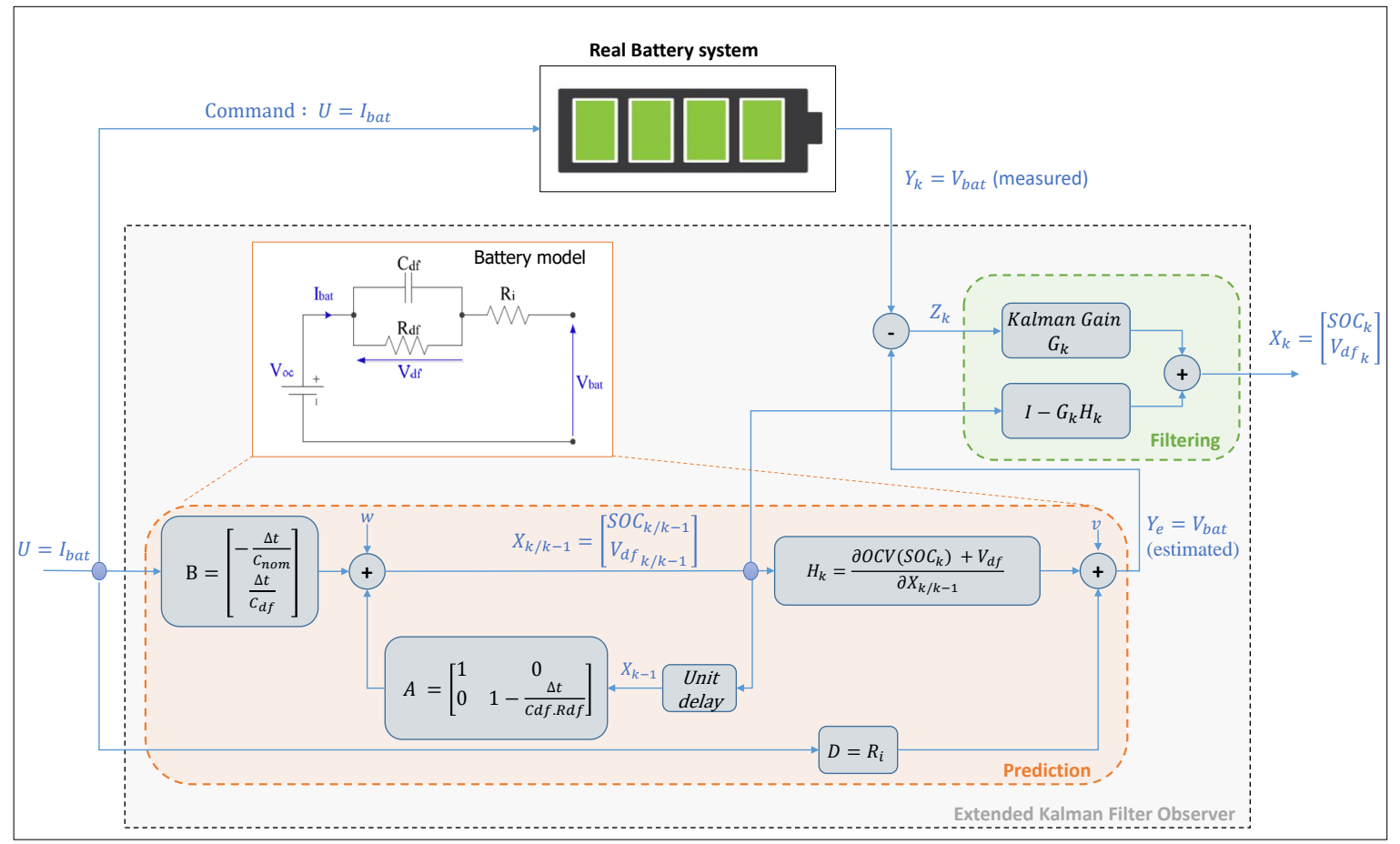

Figure 13. The principle of EKF for SOC estimation using the battery first-order model.

The considered first-order model of the Li-ion battery gives the following Equations (5) and (6).

$$
\left\{\begin{array}{l}
\dot{V}_{d f}=\frac{I_{b a t}}{C_{d f}}-\frac{V_{d f}}{C_{d f} R_{d f}}=\frac{I_{b a t}}{C_{d f}}-\frac{V_{d f}}{\tau_{d f}} \\
V_{b a t}=V_{O c}-V_{d f}-I_{b a t} R_{i}
\end{array}\right.
$$

The discretization of Equation (5) gives the relation between the actual and previous $V_{d f}$ values as in Equation (7).

$$
V_{d f_{k}}=\frac{I_{b a t} \Delta t}{C_{d f}}+\left(1+\frac{\Delta t}{C_{d f} R_{d f}}\right) V_{d f_{k-1}}
$$

The SOC is given by the Coulomb-counting formula in Equation (8) that defines it as a time integration of the battery current. The discrete form of SOC is stated in Equation (9).

$$
\begin{gathered}
S O C=S O C_{0}+\frac{-1}{C_{n o m}} \int_{t}^{t+\Delta t} I_{b a t} \Delta t \\
S O C_{k}=S O C_{k-1}-\frac{I_{\text {bat }} \Delta t}{C_{\text {nom }}}
\end{gathered}
$$


We define then the state vector composed by the battery SOC and the diffusion voltage $V_{d f}$.

We obtain then Equations (10) and (11) that define the battery model system formulation, to be solved according to Algorithm 1 and Figure 13.

$$
\left\{\begin{array}{l}
{\left[\begin{array}{c}
S O C_{k+1} \\
V_{d f_{k+1}}
\end{array}\right]=\left[\begin{array}{cc}
1 & 0 \\
0 & 1-\frac{\Delta t}{C_{d f} R_{d f}}
\end{array}\right]\left[\begin{array}{c}
S O C_{k} \\
V_{d f_{k}}
\end{array}\right]+\left[\begin{array}{c}
-\frac{\Delta t}{C_{n o m}} \\
\frac{\Delta t}{C_{d f}}
\end{array}\right] I_{b a t}} \\
V_{b a t}=O C V\left(S O C_{k}\right)-R_{i} I_{b a t}-V_{d f}
\end{array}\right.
$$

One of the best advantages of the Kalman filtering is to overcome the sensibility of the SOC estimation to the initial $S O C_{0}$. In fact, thanks to the prediction and the correction processes of the Kalman filtering algorithm, the latter allows converging to the real value of SOC even if the initial value is very far from the real one, and this according to many works [38,46,49].

Applying the EKF to the battery system proves this and can be shown in Figure 14. Assuming that we are estimating the SOC of the cell full charge from $0 \%-100 \%$, we used four different $S O C_{0}$, and we observed that the algorithm converges and gives a reliable estimation; indeed, the closer the initial random $S O C_{0}$ is to the real initial SOC, the faster the algorithm is converging.

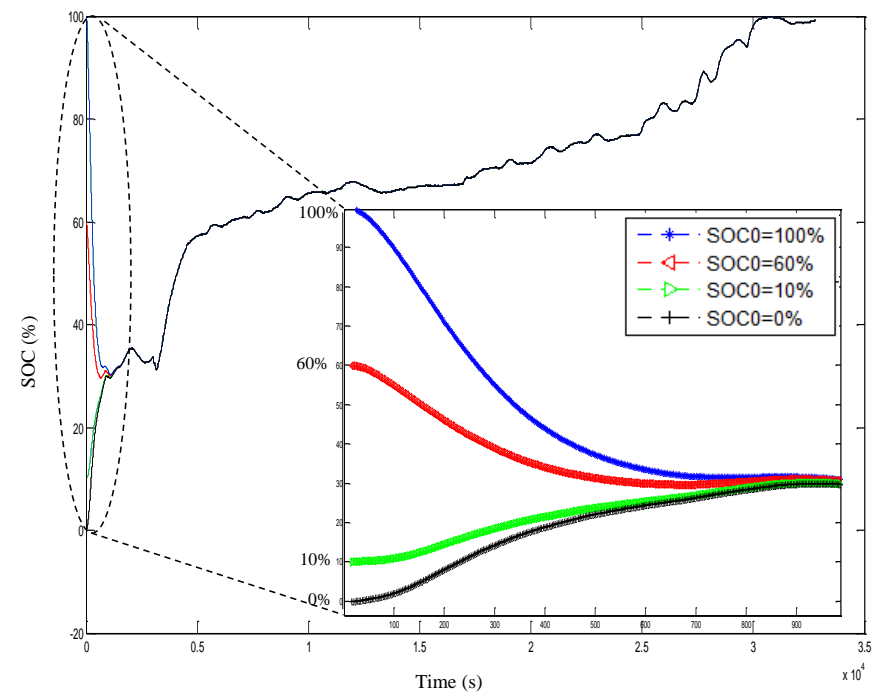

Figure 14. EKF algorithm convergence with random $S O C_{0}$.

\subsection{Experimental Results}

To evaluate the effectiveness of the implementation of our proposed OCV model for SOC estimation, the EKF is used with the five OCV models studied in this paper to compare their performances. As a reference, we used the SOC estimation given by the Coulomb-counting where the OCV-SOC experimental values are stored in a look-up table, and the relationship is given by linear regression. This reference is considered as the true SOC to be compared to EKF estimation using the different studied OCV models. Accordingly, we define two cases of current load profiles that represent the solicitation of our LEV battery pack.

- Case 1, continuous load profile:

At first, we used continuous current profile load at one cycle of charge/discharge. The simulation of EKF with different studied OCV models is shown in Figure 15.

The precision of the SOC estimation is calculated in comparison to the reference curve. The results corresponding to the estimation error of SOC percentage are presented in the following Table 9. 
As mentioned in this table, the average error given by the proposed model during one cycle of estimation by the EKF is $0.05 \%$ of SOC, which is far better than other models.

Table 9. EKF estimation error (constant current load) at $25^{\circ} \mathrm{C}$.

\begin{tabular}{cccccc}
\hline Performances & Model 1 & Model 2 & Model 3 & Model 4 & Model 5 (Proposed) \\
\hline Average error & $7.3042 \%$ & $2.5802 \%$ & $1.4177 \%$ & $1.6320 \%$ & $0.0502 \%$ \\
Maximum error & $15.4188 \%$ & $3.7622 \%$ & $2.5991 \%$ & $2.8136 \%$ & $0.0676 \%$ \\
Speed (s) & 2.90 & 13.72 & 17.62 & 11.19 & 11.68 \\
\hline
\end{tabular}

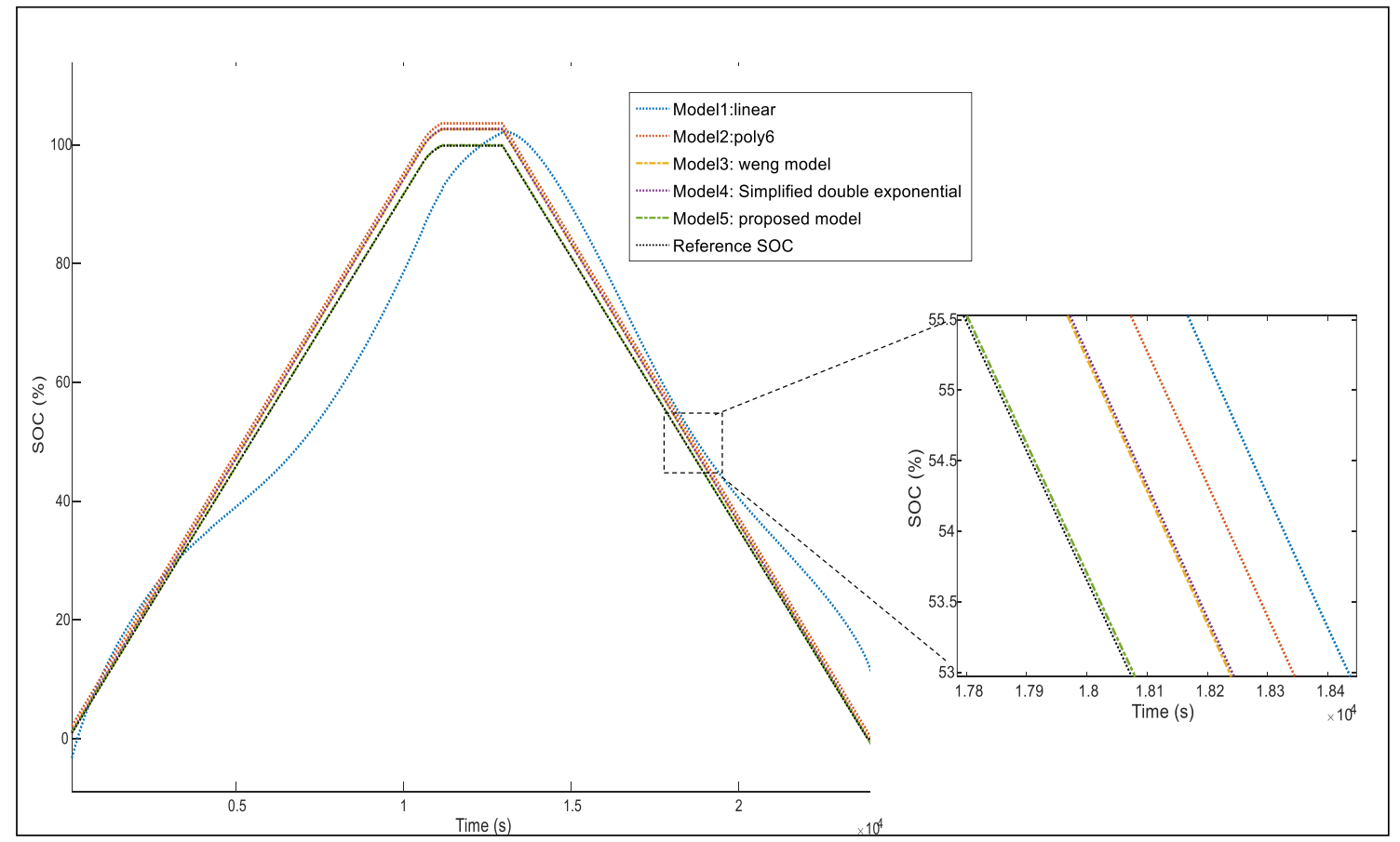

Figure 15. EKF simulation using different OCV models (constant current load) at $25^{\circ} \mathrm{C}$.

- Case 2, dynamic load profile:

Another case is considered when the load applied in the discharge is a pulsed current draw, which is correlated with the successive acceleration and deceleration of an LEV. In fact, we used dynamic discharge profile data, which simulate that type of load; then, the SOC estimation was observed. Again, the proposed OCV model gives a minor error estimation compared to other models. In fact, the estimation error is about $3 \%$ as the average value, almost twice as precise as when the OCV model is polynomial. These results are well illustrated in Figure 16 and Table 10.

As seen in Table 10, estimating SOC using our enhanced OCV model takes less time than using other models, except for the linear model, which is the fastest, and this due to the small number of coefficients and the lack of the need for linearization at each iteration of the EKF algorithm.

Table 10. EKF estimation error using different OCV models (pulse current load) at $25^{\circ} \mathrm{C}$.

\begin{tabular}{cccccc}
\hline Performances & Model 1 & Model 2 & Model 3 & Model 4 & Model 5 (Proposed) \\
\hline Average error & $9.580 \%$ & $5.725 \%$ & $4.735 \%$ & $4.776 \%$ & $2.984 \%$ \\
Maximum error & $37.068 \%$ & $7.728 \%$ & $6.739 \%$ & $6.780 \%$ & $4.604 \%$ \\
Speed (s) & 5.8 & 56.71 & 64.51 & 52.175 & 47.678 \\
\hline
\end{tabular}




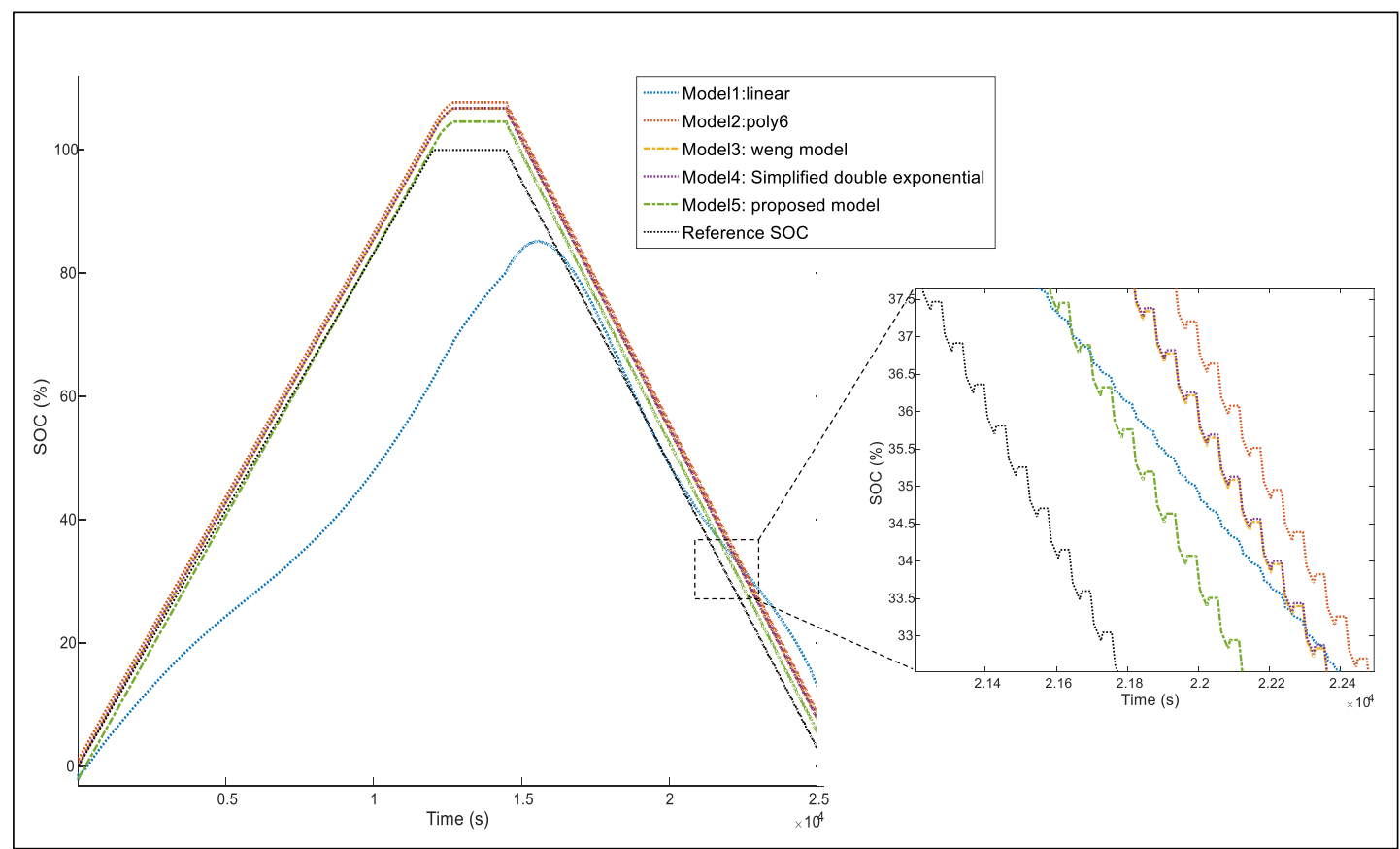

Figure 16. EKF simulation with different OCV models (pulse current load) at $25^{\circ} \mathrm{C}$.

To summarize all of the studied models' performances, we grouped all metrics to have a global comparison in Figure 17. In fact, Model 5, which is the proposed model, presents the best compromise. This OCV model is very reliable from a precision point of view, since it has the best fit, and the RMSE is about $0.01 \mathrm{~V}$; and also the best SOC estimation precision since the error is almost about $3 \%$. Additionally, from an implementation point of view, it is also the most adequate.

Actually, the number of coefficients is only five, which is far less than 12 in the Weng model, and also, it makes the EKF faster than the other models were.

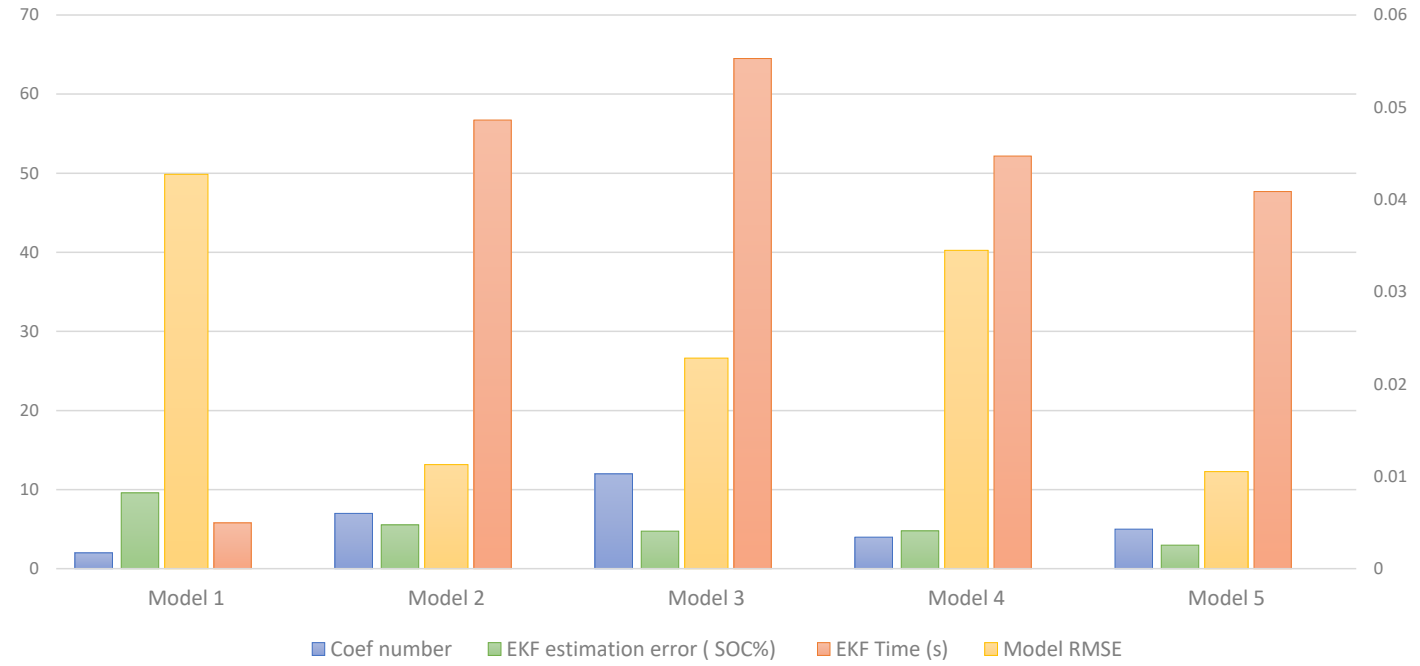

Figure 17. Comparison of the studied OCV models' performances at $25{ }^{\circ} \mathrm{C}$. 
All of these performances are proven at $25^{\circ} \mathrm{C}$, but our battery in the LEV could be exposed to heat or cold. Thus, a validation of our model should be performed to prove its reliability at a large margin of temperatures.

In fact, when applied to different pulsed profiles at various temperatures $\left(5,15,25,45^{\circ} \mathrm{C}\right)$, the algorithm shows a good accuracy reported in Table 11, where the EKF error precision compared to the real SOC given by the Coulomb-counting is almost $3 \%$ as an average value. All of the experimental results are illustrated in Figure 18.

Table 11. EKF estimation error using the proposed OCV model at different temperatures.

\begin{tabular}{ccccc}
\hline EKF SOC Estimation Error & $\mathbf{5}^{\circ} \mathbf{C}$ & $\mathbf{1 5}{ }^{\circ} \mathbf{C}$ & $\mathbf{2 5}{ }^{\circ} \mathbf{C}$ & $\mathbf{4 5}{ }^{\circ} \mathbf{C}$ \\
\hline Average error & $3.1 \%$ & $2.4 \%$ & $2.98 \%$ & $2.9 \%$ \\
Maximum error & $4.53 \%$ & $2.91 \%$ & $4.60 \%$ & $3.31 \%$ \\
\hline
\end{tabular}
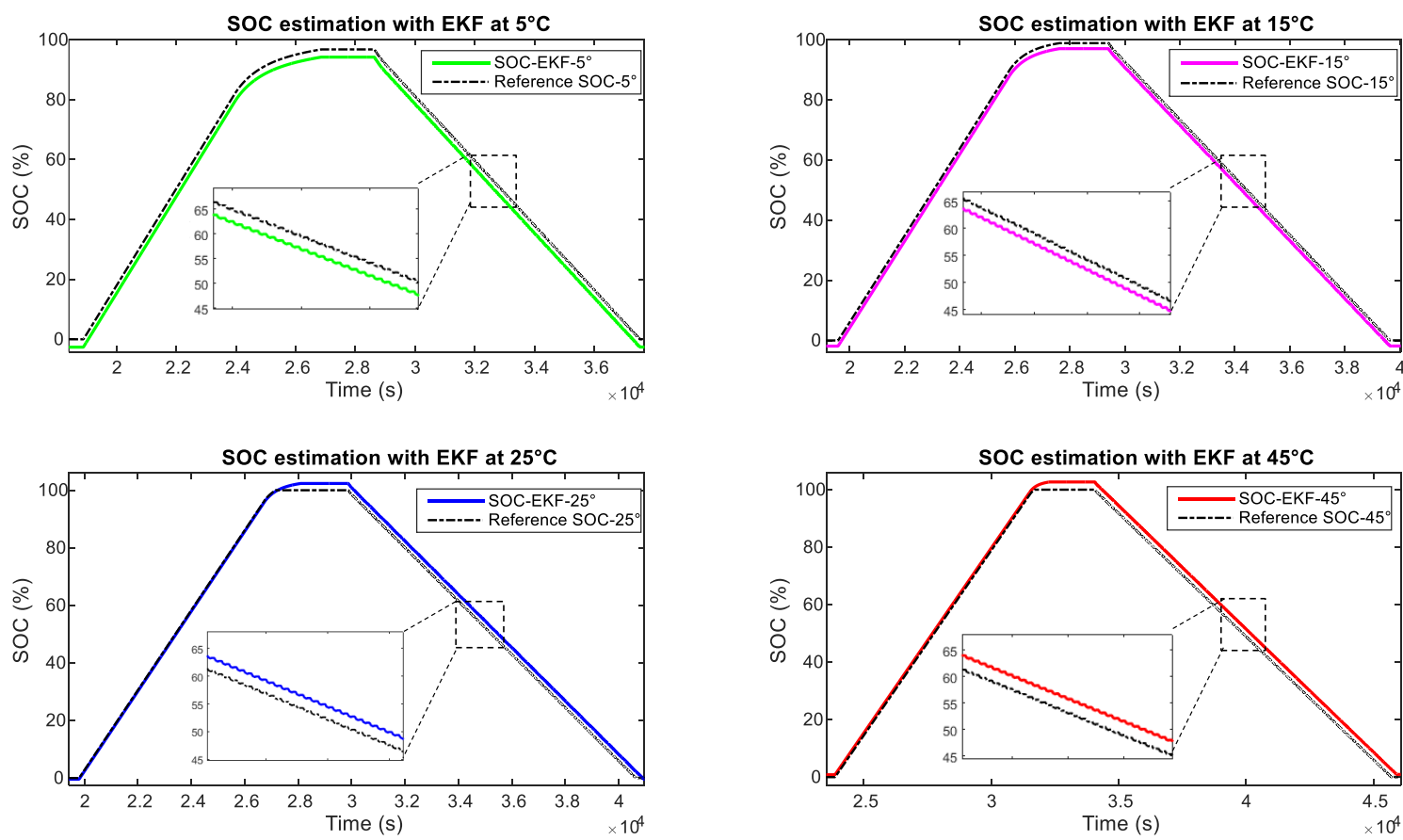

Figure 18. EKF estimation using the proposed OCV model at different temperatures.

Table 12 allows us to situate our proposed approach with the other existing approaches in the literature. Thus, we remark that our OCV model gives a good accuracy in SOC estimation by EKF using only the first-order model that integrates our new OCV model as an NMC battery equivalent model.

Table 12. Review of model-based SOC estimation approaches' performances for NMC batteries at $25^{\circ}$.

\begin{tabular}{ccccc}
\hline Ref. & OCV Model & Battery Model & SOC Algorithm & SOC Error \\
\hline Yuan et al. 2013 [49] & Polynomial & 2nd order & EKF Observer & $5 \%$ \\
Weng et al. 2014 [22] & Weng Model & 1st order & EKF & $5 \%$ \\
Zou et al. 2014 [53] & Linear & 1st order & Luenberger Observer & $2 \%$ \\
Zhang et al. 2016 [20] & combined Model & 1st order & PI Observer & $5 \%$ \\
Tran et al. 2017 [33] & Exponential & 1st order & Dual EKF & $5 \%$ \\
Baccouche et al. 2017 & Proposed Model & 1st order & EKF & $3 \%$ \\
\hline
\end{tabular}


Actually, a review of various works proposing model-based methods for the adaptive SOC estimation of the NMC battery by a state observer such as EKF, PI or Luenberger using a variety of OCV models incorporated generally in the Thevenin battery equivalent model have reached an average precision of SOC estimation that is within the margin of $5 \%$. On the other hand, using our proposed analytical OCV model, composed of the double exponential and a simple quadratic term containing only five coefficients, increases the precision of EKF estimation and reduces the error up to only $3 \%$. This improvement is due to the fact that the precision of the OCV model itself has increased, which has been projected on the SOC estimation performance.

\section{Conclusions}

In this paper, we have proposed an improved OCV model of an NMC battery. The suggested model has been beneficial to reduce the computation and memory cost in order to be implemented on the BMS of an LEV. The effectiveness of the model has been proven and compared with other existing models studied in this paper. Our model has the advantage of being more suitable for an online SOC estimation since it has less parameters than other models, a better fit with the experimental data and simplified functions. The proposed model has been used with a battery first-order model that takes into account the OCV hysteresis to estimate the SOC. We used the EKF algorithm since a local linearization of the OCV equation is needed. The experimental results when applying the EKF algorithm combined with our OCV model on both static and dynamic loads have shown a better estimation accuracy than other model-based approaches existing in the literature, which gives an error estimation of $5 \%$. In fact, the error estimation recorded by our approach is only about $3 \%$ of the SOC as an average value. All of the experiments have been reproduced at $5{ }^{\circ} \mathrm{C}, 15^{\circ} \mathrm{C}, 25^{\circ} \mathrm{C}$ and $45^{\circ} \mathrm{C}$, and the model has been proven to be useful at a large margin of temperatures. As future work, we aim to generalize this model by including both SOC and temperature as parameters in the OCV model and to improve the SOC estimation accuracy by using a higher order battery model.

Acknowledgments: This work is part of a Ph.D. thesis supported by the Project for support of the Research and Innovation System (PASRI-MOBIDOC) , IntelliBatteries company and -Laboratory of Advanced technology and Intelligent Systems (LATIS) . All of the characterization tests were done at the Mobility, Logistics and Automotive Technology Research Centre (MOBI) at the Vrije Universiteit Brussel(VUB)in Belgium. We would like to thank the MOBI team for their collaboration.

Author Contributions: Ines Baccouche analyzed the experiments, proposed the OCV model, developed the algorithm and wrote the paper. Sabeur Jemmali contributed to validating the model and editing the manuscript. Bilal Manai contributed to designing the model and editing the manuscript. Omar Noshin contributed to providing the data and editing the manuscript. Najoua Essoukri Ben Amara contributed to supervising the research project and editing the manuscript.

Conflicts of Interest: The authors declare no conflict of interest.

\section{Abbreviations}

The following abbreviations are used in this manuscript:

$\begin{array}{ll}\text { OCV } & \text { Open circuit voltage } \\ \text { SOC } & \text { State of charge } \\ \text { NMC } & \text { Nickel manganese cobalt } \\ \text { BMS } & \text { Battery management system } \\ \text { EKF } & \text { Extended Kalman filter } \\ \text { RC } & \text { resistor-capacitor } \\ \text { LEV } & \text { Light electric vehicle } \\ \text { BEV } & \text { Battery electric vehicle } \\ \text { RMSE } & \text { Root mean square error } \\ \text { DDP } & \text { Dynamic discharge pulse } \\ \text { PI observer } & \text { Proportional integral observer }\end{array}$




\section{References}

1. Reddy, T. Linden's Handbook of Batteries, 4th ed.; McGraw-Hill Education: New York, NY, USA, 2010.

2. Tarascon, J.M.; Armand, M. Issues and challenges facing rechargeable lithium batteries. Nature 2001, 414, 359-367.

3. Buchmann, I. Batteries in a Portable World: A Handbook on Rechargeable Batteries for Non-Engineers, 4th ed.; Cadex Electronics Inc.: Richmond, BC, Canada, 2016.

4. Lu, L.; Han, X.; Li, J.; Hua, J.; Ouyang, M. A review on the key issues for lithium-ion battery management in electric vehicles. J. Power Sources 2013, 226, 272-288.

5. Cheng, K.W.E.; Divakar, B.P.; Wu, H.; Ding, K.; Ho, H.F. Battery-Management System (BMS) and SOC Development for Electrical Vehicles. IEEE Trans. Veh. Technol. 2011, 60, 76-88.

6. Bergveld, H.J.; Kruijt, W.S.; Notten, P.H.L. Battery Management Systems. In Battery Management Systems: Design by Modelling; Springer: Dordrecht, The Netherlands, 2002; pp. 9-30.

7. Lewis, I.; Pierce, W. Battery Management System and Method. U.S. Patent 9,440,544, 13 September 2016.

8. Chang, W.Y. The state of charge estimating methods for battery: A review. ISRN Appl. Math. 2013, 2013, 953792.

9. Waag, W.; Fleischer, C.; Sauer, D.U. Critical review of the methods for monitoring of lithium-ion batteries in electric and hybrid vehicles. J. Power Sources 2014, 258, 321-339.

10. Chaturvedi, N.A.; Klein, R.; Christensen, J.; Ahmed, J.; Kojic, A. Algorithms for Advanced BatteryManagement Systems. IEEE Control Syst. 2010, 30, 49-68.

11. Moura, S.J.; Krstic, M.; Chaturvedi, N.A. Adaptive PDE observer for battery SOC/SOH estimation. In Proceedings of the ASME 2012 5th Annual Dynamic Systems and Control Conference joint with the JSME 2012 11th Motion and Vibration Conference, Fort Lauderdale, FL, USA, 17-19 October 2012; pp. 101-110.

12. Awadallah, M.A.; Venkatesh, B. Accuracy improvement of $\{\mathrm{SOC}\}$ estimation in lithium-ion batteries. J. Energy Storage 2016, 6, 95-104.

13. Dong, G.; Zhang, X.; Zhang, C.; Chen, Z. A method for state of energy estimation of lithium-ion batteries based on neural network model. Energy 2015, 90, 879-888.

14. Ng, K.S.; Moo, C.S.; Chen, Y.P.; Hsieh, Y.C. Enhanced coulomb counting method for estimating state-of-charge and state-of-health of lithium-ion batteries. Appl. Energy 2009, 86, 1506-1511.

15. Baccouche, I.; Mlayah, A.; Jemmali, S.; Manai, B.; Essoukri Ben Amara, N. Implementation of a Coulomb counting algorithm for SOC estimation of Li-Ion battery for multimedia applications. In Proceedings of the 2015 IEEE 12th International Multi-Conference on Systems, Signals Devices (SSD15), Sakiet Ezzit Sfax, Tunisia, 16-19 March 2015; pp. 1-6.

16. Jeong, Y.M.; Cho, Y.K.; Ahn, J.H.; Ryu, S.H.; Lee, B.K. Enhanced Coulomb counting method with adaptive SOC reset time for estimating OCV. In Proceedings of the 2014 IEEE Energy Conversion Congress and Exposition (ECCE), Pittsburgh, PA, USA, 14-18 September 2014; pp. 1313-1318.

17. Nugroho, A.; Rijanto, E.; Wijaya, F.D.; Nugroho, P. Battery state of charge estimation by using a combination of Coulomb Counting and dynamic model with adjusted gain. In Proceedings of the 2015 International Conference on Sustainable Energy Engineering and Application (ICSEEA), Bandung, Indonesia, 5-7 October 2015; pp. 54-58.

18. Tian, Y.; Li, D.; Tian, J.; Xia, B. A comparative study of state-of-charge estimation algorithms for lithium-ion batteries in wireless charging electric vehicles. In Proceedings of the 2016 IEEE PELS Workshop on Emerging Technologies: Wireless Power Transfer (WoW), Knoxville, TN, USA, 4-6 October 2016; pp. 186-190.

19. He, H.; Xiong, R.; Zhang, X.; Sun, F.; Fan, J. State-of-Charge Estimation of the Lithium-Ion Battery Using an Adaptive Extended Kalman Filter Based on an Improved Thevenin Model. IEEE Trans. Veh. Technol. 2011, 60, 1461-1469.

20. Zhang, C.; Jiang, J.; Zhang, L.; Liu, S.; Wang, L.; Loh, P.C. A Generalized SOC-OCV Model for Lithium-Ion Batteries and the SOC Estimation for LNMCO Battery. Energies 2016, 9, 900.

21. Wang, Q.; Wang, J.; Zhao, P.; Kang, J.; Yan, F.; Du, C. Correlation between the model accuracy and model-based \{SOC\} estimation. Electrochim. Acta 2017, 228, 146-159.

22. Weng, C.; Sun, J.; Peng, H. A unified open-circuit-voltage model of lithium-ion batteries for state-of-charge estimation and state-of-health monitoring. J. Power Sources 2014, 258, 228-237. 
23. Nikolian, A.; Firouz, Y.; Gopalakrishnan, R.; Timmermans, J.M.; Omar, N.; van den Bossche, P.; van Mierlo, J. Lithium ion batteries-Development of advanced electrical equivalent circuit models for nickel manganese cobalt lithium-ion. Energies 2016, 9, 360.

24. Ashwin, T.; McGordon, A.; Jennings, P. Electrochemical modeling of Li-ion battery pack with constant voltage cycling. J. Power Sources 2017, 341, 327-339.

25. Sun, K.; Shu, Q. Overview of the types of battery models. In Proceedings of the IEEE 2011 30th Chinese Control Conference (CCC), Yantai, China, 22-24 July 2011; pp. 3644-3648.

26. Ghossein, N.E.; Salameh, J.P.; Karami, N.; Hassan, M.E.; Najjar, M.B. Survey on electrical modeling methods applied on different battery types. In Proceedings of the 2015 Third International Conference on Technological Advances in Electrical, Electronics and Computer Engineering (TAEECE), Beirut, Lebanon, 29 April-1 May 2015; pp. 39-44.

27. Omar, N.; Widanage, D.; Abdel Monem, M.; Firouz, Y.; Hegazy, O.; Van den Bossche, P.; Coosemans, T.; Van Mierlo, J. Optimization of an advanced battery model parameter minimization tool and development of a novel electrical model for lithium-ion batteries. Int. Trans. Electr. Energy Syst. 2014, 24, 1747-1767.

28. Hua, Y.; Xu, M.; Li, M.; Ma, C.; Zhao, C. Estimation of State of Charge for Two Types of Lithium-Ion Batteries by Nonlinear Predictive Filter for Electric Vehicles. Energies 2015, 8, 3556-3577.

29. Roscher, M.A.; Bohlen, O.; Vetter, J. OCV hysteresis in Li-ion batteries including two-phase transition materials. Int. J. Electrochem. 2011, 2011, 984320.

30. Kim, J.; Seo, G.S.; Chun, C.; Cho, B.H.; Lee, S. OCV hysteresis effect-based SOC estimation in extended Kalman filter algorithm for a LiFePO4/C cell. In Proceedings of the 2012 IEEE International Electric Vehicle Conference, Greenville, SC, USA, 4-8 March 2012; pp. 1-5.

31. Xing, Y.; He, W.; Pecht, M.; Tsui, K.L. State of charge estimation of lithium-ion batteries using the open-circuit voltage at various ambient temperatures. Appl. Energy 2014, 113, 106-115.

32. Zhang, C.; Li, K.; Pei, L.; Zhu, C. An integrated approach for real-time model-based state-of-charge estimation of lithium-ion batteries. J. Power Sources 2015, 283, 24-36.

33. Tran, N.T.; Khan, A.B.; Choi, W. State of Charge and State of Health Estimation of AGM VRLA Batteries by Employing a Dual Extended Kalman Filter and an ARX Model for Online Parameter Estimation. Energies 2017, 10, 137.

34. Hu, X.; Li, S.; Peng, H.; Sun, F. Robustness analysis of State-of-Charge estimation methods for two types of Li-ion batteries. J. Power Sources 2012, 217, $209-219$.

35. Plett, G.L. Extended Kalman filtering for battery management systems of LiPB-based $\{$ HEV $\}$ battery packs: Part 2. Modeling and identification. J. Power Sources 2004, 134, 262-276.

36. Widanage, W.; Barai, A.; Chouchelamane, G.; Uddin, K.; McGordon, A.; Marco, J.; Jennings, P. Design and use of multisine signals for Li-ion battery equivalent circuit modeling. Part 2: Model estimation. J. Power Sources 2016, 324, 61-69.

37. Luo, Z.; Li, Y.; Lou, Y. An adaptive Kalman filter to estimate state-of-charge of lithium-ion batteries. In Proceedings of the 2015 IEEE International Conference on Information and Automation, Lijiang, China , 8-10 August 2015; pp. 1227-1232.

38. Baccouche, I.; Jemmali, S.; Manai, B.; Chaibi, R.; Essoukri Ben Amara, N. Hardware implementation of an algorithm based on Kalman filtrer for monitoring low capacity Li-ion batteries. In Proceedings of the 2016 7th International Renewable Energy Congress (IREC), Hammamet, Tunisia, 22-24 March 2016, pp. 1-6.

39. Szumanowski, A.; Chang, Y. Battery Management System Based on Battery Nonlinear Dynamics Modeling. IEEE Trans. Veh. Technol. 2008, 57, 1425-1432.

40. Chen, M.; Rincon-Mora, G.A. Accurate electrical battery model capable of predicting runtime and I-V performance. IEEE Trans. Energy Conversion 2006, 21, 504-511.

41. Hu, Y.; Yurkovich, S.; Guezennec, Y.; Yurkovich, B. Electro-thermal battery model identification for automotive applications. J. Power Sources 2011, 196, $449-457$.

42. Neumann, D.; Lichte, S. A multidimensional battery discharge model with thermal feedback applied to a lithium-ion battery pack. In Proceedings of the NDIA Ground Vehicle Systems Engineering and Technology Symposium, Dearborn, MI, USA, 10-11 August 2011.

43. Pattipati, B.; Balasingam, B.; Avvari, G.; Pattipati, K.; Bar-Shalom, Y. Open circuit voltage characterization of lithium-ion batteries. J. Power Sources 2014, 269, 317-333. 
44. Goutam, S.; Timmermans, J.M.; Omar, N.; Bossche, P.V.D.; Van Mierlo, J. Comparative Study of Surface Temperature Behavior of Commercial Li-Ion Pouch Cells of Different Chemistries and Capacities by Infrared Thermography. Energies 2015, 8, 8175-8192.

45. Cicconi, P.; Landi, D.; Germani, M. Thermal analysis and simulation of a Li-ion battery pack for a lightweight commercial \{EV\}. Appl. Energy 2017, 192, 159-177.

46. Huria, T.; Ceraolo, M.; Gazzarri, J.; Jackey, R. Simplified Extended Kalman Filter Observer for SOC Estimation of Commercial Power-Oriented LFP Lithium Battery Cells; SAE Technical Paper 2013-01-1544; SAE International: Warrendale, PA, USA, 2013.

47. Tjandra, R.; Tseng, K.J.; Thanagasundram, S.; Jossen, A. State of charge estimation considering OCV hysteresis in lithium iron phosphate battery for UPS applications. In Proceedings of the 2015 IEEE International Telecommunications Energy Conference (INTELEC), Osaka, Japan, 18-22 October 2015; pp. 1-6.

48. Cho, I.; Kim, D. Method of Setting Initial Value of SOC of Battery Using OCV Hysteresis Depending on Temperatures. U.S. Patent App. 11/370,403, 14 September 2006.

49. Yuan, S.; Wu, H.; Yin, C. State of Charge Estimation Using the Extended Kalman Filter for Battery Management Systems Based on the ARX Battery Model. Energies 2013, 6, 444-470.

50. Zhang, W.; Shi, W.; Ma, Z. Adaptive unscented Kalman filter based state of energy and power capability estimation approach for lithium-ion battery. J. Power Sources 2015, 289, 50 - 62.

51. Plett, G.L. Sigma-point Kalman filtering for battery management systems of LiPB-based $\{\mathrm{HEV}\}$ battery packs: Part 2: Simultaneous state and parameter estimation. J. Power Sources 2006, 161, 1369-1384.

52. Yu, Z.; Huai, R.; Xiao, L. State-of-Charge Estimation for Lithium-Ion Batteries Using a Kalman Filter Based on Local Linearization. Energies 2015, 8, 7854-7873.

53. Zou, Z.; Xu, J.; Mi, C.; Cao, B.; Chen, Z. Evaluation of Model Based State of Charge Estimation Methods for Lithium-Ion Batteries. Energies 2014, 7, 5065-5082.

(C) 2017 by the authors. Licensee MDPI, Basel, Switzerland. This article is an open access article distributed under the terms and conditions of the Creative Commons Attribution (CC BY) license (http://creativecommons.org/licenses/by/4.0/). 